\title{
REVISED BED: a Biological Entity Dictionary based on a graph
}

\section{data model [version 3; peer review: 2 approved]}

\author{
Patrice Godard (D1,2, Jonathan van Eyll2 \\ ${ }^{1}$ Clarivate Analytics, Carlsbad, CA, 92008, USA \\ 2UCB Pharma, Braine-l'Alleud, 1420, Belgium
}

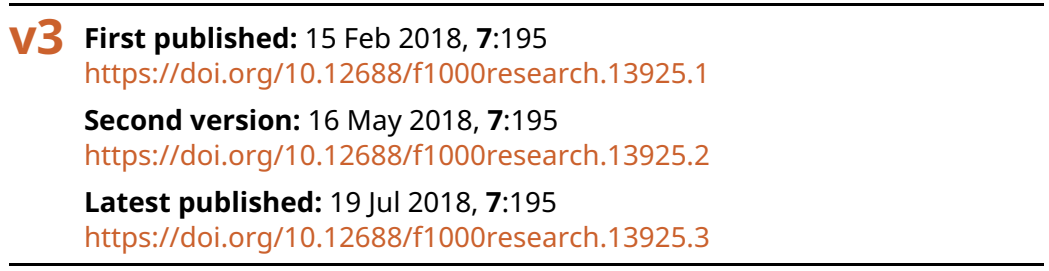

\section{Abstract}

The understanding of molecular processes involved in a specific biological system can be significantly improved by combining and comparing different data sets and knowledge resources. However, these information sources often use different identification systems and an identifier conversion step is required before any integration effort. Mapping between identifiers is often provided by the reference information resources and several tools have been implemented to simplify their use. However, most of these tools do not combine the information provided by individual resources to increase the completeness of the mapping process. Also, deprecated identifiers from former versions of databases are not taken into account. Finally, finding automatically the most relevant path to map identifiers from one scope to the other is often not trivial. The Biological Entity Dictionary (BED) addresses these three challenges by relying on a graph data model describing possible relationships between entities and their identifiers. This model has been implemented using Neo4j and an R package provides functions to query the graph but also to create and feed a custom instance of the database. This design combined with a local installation of the graph database and a cache system make BED very efficient to convert large lists of identifiers.

\section{Keywords}

genomics, transcriptomics, proteomics, RNA-seq, microarray, database, identifiers

\author{
Open Peer Review

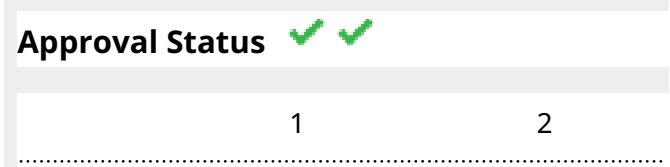 \\ version 3 \\ (revision) \\ 19 Jul 2018 \\ version 2 \\ (revision) \\ 16 May 2018 \\ version 1 \\ 15 Feb 2018

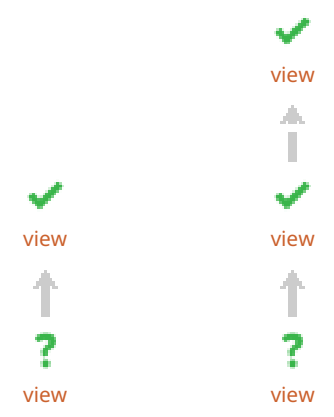 \\ 1. Denise Slenter (DD, Maastricht University, \\ Maastricht, The Netherlands \\ Martina M. Summer-Kutmon (D), \\ Maastricht University, Maastricht, The \\ Netherlands \\ 2. T. Ian Simpson ID, University of Edinburgh, \\ Edinburgh, UK
}

Any reports and responses or comments on the article can be found at the end of the article.

This article is included in the RPackage

gateway. 
Corresponding author: Patrice Godard (patrice.godard@ucb.com)

Author roles: Godard P: Conceptualization, Methodology, Software, Validation, Writing - Original Draft Preparation, Writing - Review \& Editing; van Eyll J: Supervision, Validation, Writing - Review \& Editing

Competing interests: No competing interests were disclosed.

Grant information: This work was entirely supported by UCB Pharma. The authors declared that no grants were involved in supporting this work.

Copyright: $\odot 2018$ Godard P and van Eyll J. This is an open access article distributed under the terms of the Creative Commons

Attribution License, which permits unrestricted use, distribution, and reproduction in any medium, provided the original work is properly cited. Data associated with the article are available under the terms of the Creative Commons Zero "No rights reserved" data waiver (CC0 1.0 Public domain dedication).

How to cite this article: Godard P and van Eyll J. BED: a Biological Entity Dictionary based on a graph data model [version 3; peer review: 2 approved] F1000Research 2018, 7:195 https://doi.org/10.12688/f1000research.13925.3

First published: 15 Feb 2018, 7:195 https://doi.org/10.12688/f1000research.13925.1 


\section{REVISED Amendments from Version 2}

This revision addresses minor issues raised by the referees in the former version of the article. Mainly, we have added a sentence in the Data model section explaining that deprecated identifiers are handled using "is_replaced_by" relationships. We now also provide a DOI for the specific docker image used to generate the results presented in this article.

See referee reports

\section{Introduction}

Since the advent of genome sequencing projects, many technologies have been developed to get access to different molecular information on a large scale and with high throughput. DNA micro-arrays are probably the archetype of such technology because of their historical impact on gathering data related to nucleic acids: genomic DNA and RNA. They triggered the emergence of "omics" fields of research such as genomics, epigenomics or transcriptomics. Lately massive parallel sequencing further increased the throughput of data generation related to nucleic acids by several orders of magnitude. In a different way, mass spectrometry-related technologies allow the identification and the quantification of many kinds of molecular entities such as metabolites and proteins. Many information systems have been developed to manage the exploding amount of data and knowledge related to biological molecular entities. These resources manage different aspects of the data. For example, some are genome or proteome centered, whereas others are focused on molecular interactions and pathways. Thus, all these resources rely on different identifier systems to organize concepts of their interest. The value of all the experimental data and all the knowledge collected in public or private resources is very high as such but is also often synergistically leveraged by their cross comparison in a dedicated manner. Indeed, many data sets can be relevant when addressing the understanding of a specific biological system, a phenotypic trait or a disease for example. These data sets can focus on different biological entities such as transcripts or proteins in different tissues, conditions or organisms. Comparing all these data and integrating them with available knowledge requires the ability to map the identifiers on which each resource relies.

To achieve this task public and proprietary information systems provide mapping tables between their own identifiers (ID) and those from other resources. Furthermore, many tools have been developed to facilitate the access to this information. Ensembl BioMart (Kinsella et al., 2011), mygene (Wu et al., 2013), and g:Profiler (Reimand et al., 2016a) are popular examples among many others. These resources are convenient and easy to use with information managed by their maintainers. However, in general (BioMart provides tools to configure marts), this information cannot be easily customized, optimized or extended by an empowered user according to his knowledge or to internal, non-public or non-standard data. Recognizing these challenges van Iersel et al. (2010) proposed the BridgeDb framework providing to bioinformatics developers a standard interface between tools and mapping services and also allowing the easy integration of custom data by a transitivity mechanism. However, to our knowledge, transitivity in the BridgeDb framework is not leveraged to improve the completeness of ID conversion (e.g. the number of Ensembl gene ID actually converted to Entrez gene ID).

Here we present BED: a biological entity dictionary. BED has been developed to address three main challenges. The first one is related to the completeness of identifier mappings. Indeed, direct mapping information provided by the different systems are not always complete and can be enriched by mappings provided by other resources. More interestingly, direct mappings not identified by any of these resources can be indirectly inferred by using mappings to a third reference. For example, many human Ensembl gene ID are not directly mapped to any Entrez gene ID but such mappings can be inferred using respective mappings to HGNC ID. The second challenge is related to the mapping of deprecated identifiers. Indeed, entity identifiers can change from one resource release to another. The identifier history is provided by some resources, such as Ensembl or the NCBI, but it is generally not used by mapping tools. The third challenge is related to the automation of the mapping process according to the relationships between the biological entities of interest. Indeed, mapping between gene and protein ID scopes should not be done the same way than between two scopes regarding gene ID. Also, converting identifiers from different organisms should be possible using gene orthologs information.

To meet these challenges, we designed a graph data model describing possible relationships between different biological entities and their identifiers. This data model has been implemented with the Neo4j ${ }^{\circledR}$ graph database (Neo4j inc, 2017). Graph databases are very efficient to implement biological data models. 
They are more and more used in different fields of application. For example, they are successfully used for integrating various pieces of knowledge (Pareja-Tobes et al., 2015; Yoon et al. (2017)), describing disease and phenotype relationships (Pareja-Tobes et al., 2015) or modeling molecular networks and pathways (Dai et al., 2016; Fabregat et al. (2018)).

In addition to the $\mathrm{Neo}^{\circledR} \mathrm{j}^{\circledR}$ graph database, conversion rules have been defined and coded in an $\mathrm{R}$ ( $\mathrm{R}$ Core Team, 2017) package. A particular attention has been put on the efficiency of the tool by implementing a cache system making recurrent queries fast.

Finally, we provide, for convenience, an instance of the BED database focused on human, mouse and rat organisms. Nevertheless, many functions are available in the $\mathrm{R}$ package to customize this instance or to construct other instances tailored to other needs.

\section{Methods}

Data model

The BED (Biological Entity Dictionary) system relies on a data model inspired by the central dogma of molecular biology (Crick, 1970) and describing relationships between molecular concepts usually manipulated in the frame of genomics studies (Figure 1). A biological entity identifier (BEID) can identify either a Gene (GeneID), a Transcript (TranscriptID), a Peptide (PeptideID) or an Object (ObjectID). Object entities can correspond to complex concepts coded by any number of genes (i.e. a protein complex or a molecular function). $B E I D$ are extracted from public or private databases $(B E D B)$. BEDB can provide an Attribute related to each

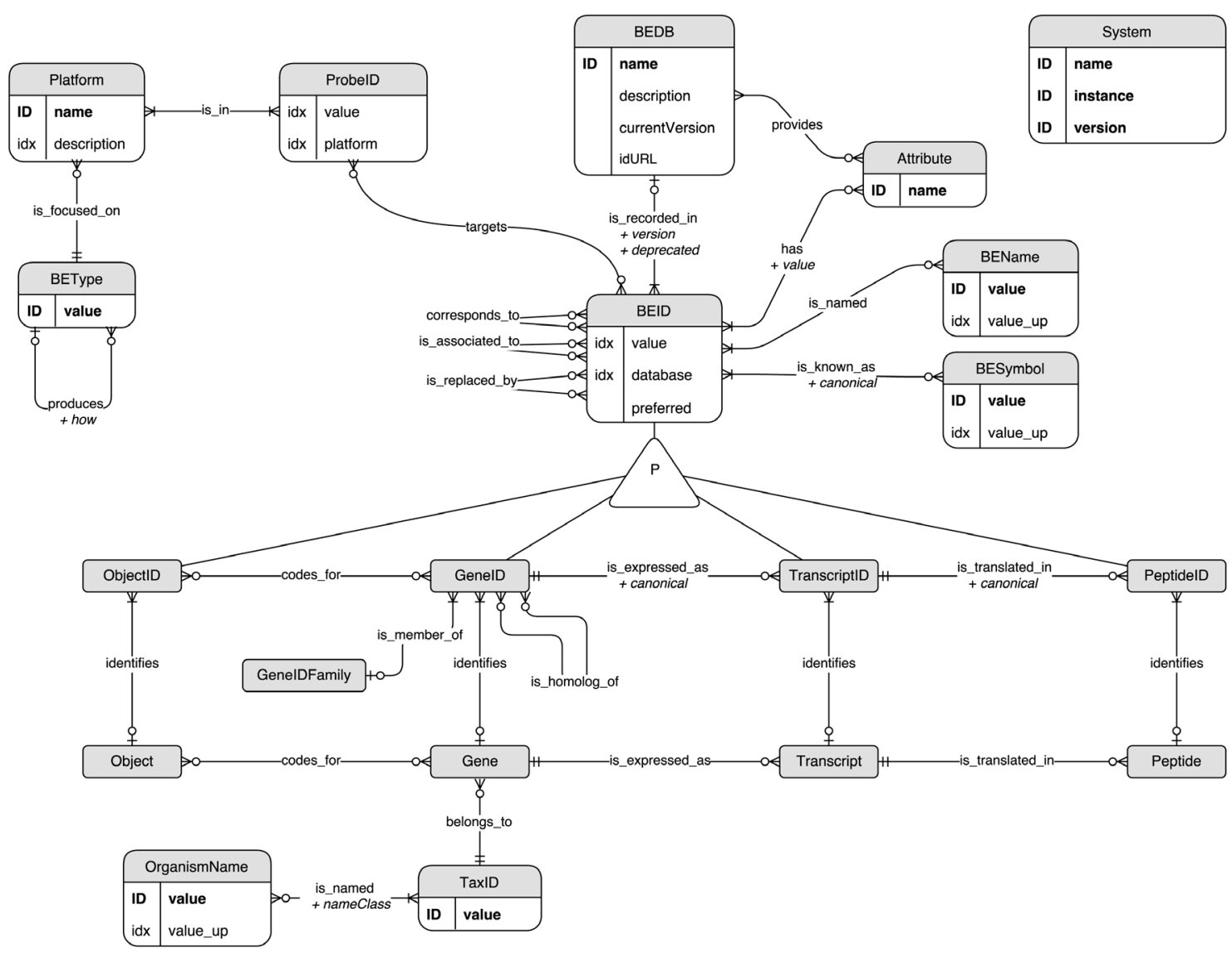

Figure 1. The BED graph data model. The model is shown as an Entity/Relationship (ER) diagram: entities correspond to graph nodes and relationships to graph edges. "ID" and "idx" indicate if the corresponding entity property is unique or indexed respectively. Some redundancies occur in this data model. Indeed some "value" properties are duplicated in upper case ("value_up") in order to improve the performance of case-insensitive searches. Also, the database of a BEID node is provided as a property to ensure uniqueness of the couples of "database" and "value" properties. The same approach has been applied for the "platform" property of ProbelD nodes. 
BEID. For example, it can be the sequencing region provided by the Ensembl database (Zerbino et al., 2018) or the identifier status provided by Uniprot (The UniProt Consortium, 2017). BEID can have one or several associated names (BENames) and symbols (BESymbol). GeneID can have one or several homologs in other organisms belonging to the same GeneIDFamily. Many genomics platforms, such as micro-arrays, allow the identification of biological entities by using probes identified by ProbeID. In general, BEID can be targeted by several probes belonging to a Platform which is focused on one, and only one, type of entity (BEType) among those described above: Gene, Transcript, Peptide or Object.

According to this data model, the scope of an identifier is defined by three features:

- its type: a BEType or "Probe"

- its source: a database for BEID or a platform for ProbeID

- the organism to which it refers

A BEType can have several BEType products but can be the product of at most one BEType. This constraint allows the unambiguous identification of the most relevant path to convert identifiers from one scope to another and is fulfilled by the current data model: peptides are only produced from transcripts, which are only produced from genes, which can also code for objects.

$B E I D$ identifying the same biological entity are related through three different kinds of relationship according to the information available in the source databases, and to the decision made by the database administrator about how to use them. Two BEID which corresponds_to each other both identify the same biological entity. A BEID which is_associated_to or which is_replaced_by another BEID does not directly identify any biological entity: the link is always indirect through one or several other BEID. Therefore, by design a BEID which is_associated_to or which is_replaced_by another BEID can be related to several different biological entities. It is not the case for other BEID which identify one and only one biological entity. Relationships corresponds_to and is_associated_to are used to map identifiers from different databases with different transitivity properties (as explained below), whereas is_replaced_by relationships are used to map deprecated identifiers from former versions of the same database. This set of possible relationships allows the indirect mapping of different identifiers not necessarily provided by any integrated resource.

\section{Implementation}

In order to efficiently leverage an indirect path through these different relationships, the data model has been implemented in a Neo4j ${ }^{\circledR}$ graph database (Neo4j inc, 2017).

Two R (R Core Team, 2017) packages have been developed to feed and query the database. The first one, neo2R, provides low level functions to interact with $\mathrm{Neo}^{\circledR} \mathrm{j}^{\circledR}$. The second $\mathrm{R}$ package, BED, provides functions to feed and query the BED Neo4j $\mathrm{j}^{\circledR}$ graph database according to the data model described above.

\section{Feeding the database}

Many functions are provided within the package to build a tailored BED database instance. These functions are not exported in order not to mislead the user when querying the database (which is the expected most frequent usage of the system). An R markdown document showing how to build a BED database instance for human, mouse and rat organisms is provided within the package. It can be adapted to other organisms or needs.

Briefly, these functions can be divided according to three main levels:

- The lowest level function is the bedImport function which loads a table in the $\mathrm{Neo}^{\circledR} \mathrm{j}^{\circledR}$ database according to a Cypher ${ }^{\circledR}$ query.

- Functions of the second level allow loading identifiers and relationships tables ensuring the integrity of the data model:

- loadBE, loadProbes, loadorganisms, loadBeAttribute, loadBEsymbols, loadBENames and loadBEVersion are used to load information about BEID and ProbeID.

- loadHistory, loadCorrespondsTo, loadIsAssociatedTo, loadIsExpressedAs, loadIsTranslatedIn, loadCodesFor and loadIsHomologof are used to load relationships existing between BEID and ProbeID. 
- Highest level functions are helpers for loading information provided by some public resources in different specific formats. The following resources are currently supported but other resources can be managed by the user by calling the functions mentioned above:

- Ensembl (Zerbino et al., 2018): getEnsemblGeneIds, getEnsemblTranscriptIds, getEnsembl PeptideIds

- NCBI (NCBI Resource Coordinators, 2017): loadNcbiTax, getNcbiGeneTransPep, loadNCBIEntrezGOFunctions

- Uniprot (The UniProt Consortium, 2017): getUniprot

- Clarivate Analytics MetaBase ${ }^{\circledR}$ (Clarivate Analytics, 2017): loadMBObjects

Available database instance

An instance of the BED database (UCB-Human), built using the script provided in the BED R package, is available in a Docker ${ }^{\circledR}$ image (Docker inc, 2017) available here: https:/hub.docker.com/r/patzaw/bed-ucb-human/ (tag 2018-04-30). This instance is focused on Homo sapiens, Mus musculus and Rattus norvegicus organisms and it has been built from these resources:

- Ensembl (Zerbino et al., 2018)

- NCBI (NCBI Resource Coordinators, 2017)

- Uniprot (The UniProt Consortium, 2017)

- biomaRt (Durinck et al., 2009)

- GEOquery (Davis \& Meltzer, 2007)

- Clarivate Analytics MetaBase ${ }^{\circledR}$ (Clarivate Analytics, 2017)

The results and the use cases described below were obtained and executed using this instance of the BED database.

The numbers of BEID available in this BED database instance and which can be mapped to each other are shown in Table 1. In total, 3,559,720 BEID are available in this BED instance. This number includes deprecated identifiers without successor and which therefore cannot be mapped to any other identifier. All the genomics platforms included in this BED database instance are shown in Table 2. They provide mapping to BEID from 354,205 ProbeID in total.

Transitivity management

In the context of mapping identifiers, transitivity is the inference of a cross-reference between $\mathrm{A}$ and $\mathrm{C}$ based on existing cross-references between A and B and between B and C. Depending on how biological entities are defined, transitivity is desirable or not. In BED the transitivity mechanism is managed by the two following relationships: corresponds_to and is_associated_to. On one hand, the corresponds_to relationships make the mapping transitive since two BEIDs which are connected through this kind of relationship are considered to identify the same BE. On the other hand, a BEID which is_associated_to another one does not automatically identify the same BE making this kind of relationship not available for transitive mappings. When the BED database is fed, the user chooses which relationship should be of type corresponds_to or of type is_associated_to. For example, in the instance described above, cross-references provided by Ensembl from Ensembl gene identifiers to Entrez, HGNC and Vega gene identifiers are considered as corresponds_to relationships whereas cross-references to miRbase, Unigene and OMIM are considered as is_associated_to relationships.

Querying the database

The $B E D \mathrm{R}$ package provides functions to connect to the Neo4j ${ }^{\circledR}$ database (connectToBed) and to directly query it (bedCall). Beside these two functions, others of higher level are provided to explore available data, to manage identifiers and to convert them from one scope to another.

Different functions can be used to explore the data model by listing the type of BE (listBe) or describing their relationships (firstCommonUpstreamBe). Available organisms, databases and platforms can also be retrieved using listorganisms, listBeIdSources and listPlatforms functions. 
Table 1. Numbers of BEID available in the BED UCB-Human database instance. Numbers have been split according to the BEType and the organism. Only BEID which can be mapped to each other are taken into account (i.e. excluding deprecated identifiers without successor)

\begin{tabular}{|c|c|c|c|c|}
\hline BE & Organism & Database & BEID & URL \\
\hline Gene & Homo sapiens & MIM_GENE & 17,215 & http://www.omim.org \\
\hline Gene & Homo sapiens & miRBase & 1,881 & http://www.mirbase.org \\
\hline Gene & Homo sapiens & UniGene & 29,237 & https://www.ncbi.nlm.nih.gov \\
\hline Gene & Homo sapiens & Ens_gene & 69,056 & http://www.ensembl.org \\
\hline Gene & Homo sapiens & HGNC & 41,233 & http://www.genenames.org \\
\hline Gene & Homo sapiens & EntrezGene & 81,684 & https://www.ncbi.nlm.nih.gov \\
\hline Gene & Homo sapiens & Vega_gene & 19,141 & http://vega.sanger.ac.uk \\
\hline Gene & Homo sapiens & MetaBase_gene & 23,356 & https://portal.genego.com \\
\hline Gene & Mus musculus & miRBase & 1,193 & http://www.mirbase.org \\
\hline Gene & Mus musculus & UniGene & 29,826 & https://www.ncbi.nlm.nih.gov \\
\hline Gene & Mus musculus & Ens_gene & 57,601 & http://www.ensembl.org \\
\hline Gene & Mus musculus & MGI & 80,387 & http://www.informatics.jax.org \\
\hline Gene & Mus musculus & EntrezGene & 103,570 & https://www.ncbi.nlm.nih.gov \\
\hline Gene & Mus musculus & Vega_gene & 18,163 & http://vega.sanger.ac.uk \\
\hline Gene & Mus musculus & MetaBase_gene & 20,628 & https://portal.genego.com \\
\hline Gene & Rattus norvegicus & miRBase & 495 & http://www.mirbase.org \\
\hline Gene & Rattus norvegicus & UniGene & 18,570 & https://www.ncbi.nlm.nih.gov \\
\hline Gene & Rattus norvegicus & Ens_gene & 34,963 & http://www.ensembl.org \\
\hline Gene & Rattus norvegicus & RGD & 46,973 & https://rgd.mcw.edu \\
\hline Gene & Rattus norvegicus & EntrezGene & 57,026 & https://www.ncbi.nlm.nih.gov \\
\hline Gene & Rattus norvegicus & Vega_gene & 1,146 & http://vega.sanger.ac.uk \\
\hline Gene & Rattus norvegicus & MetaBase_gene & 17,505 & https://portal.genego.com \\
\hline Transcript & Homo sapiens & Ens_transcript & 233,600 & http://www.ensembl.org \\
\hline Transcript & Homo sapiens & Vega_transcript & 34,302 & http://vega.sanger.ac.uk \\
\hline Transcript & Homo sapiens & RefSeq & 175,183 & https://www.ncbi.nlm.nih.gov \\
\hline Transcript & Mus musculus & Ens_transcript & 139,040 & http://www.ensembl.org \\
\hline Transcript & Mus musculus & Vega_transcript & 25,704 & http://vega.sanger.ac.uk \\
\hline Transcript & Mus musculus & RefSeq & 114,509 & https://www.ncbi.nlm.nih.gov \\
\hline Transcript & Rattus norvegicus & Ens_transcript & 42,393 & http://www.ensembl.org \\
\hline Transcript & Rattus norvegicus & Vega_transcript & 1,271 & http://vega.sanger.ac.uk \\
\hline Transcript & Rattus norvegicus & RefSeq & 97,882 & https://www.ncbi.nlm.nih.gov \\
\hline Peptide & Homo sapiens & Ens_translation & 112,939 & http://www.ensembl.org \\
\hline Peptide & Homo sapiens & Vega_translation & 35,332 & http://vega.sanger.ac.uk \\
\hline Peptide & Homo sapiens & RefSeq_peptide & 121,813 & https://www.ncbi.nlm.nih.gov \\
\hline Peptide & Homo sapiens & Uniprot & 233,935 & http://www.uniprot.org \\
\hline Peptide & Mus musculus & Ens_translation & 66,352 & http://www.ensembl.org \\
\hline Peptide & Mus musculus & Vega_translation & 26,386 & http://vega.sanger.ac.uk \\
\hline Peptide & Mus musculus & RefSeq_peptide & 81,089 & https://www.ncbi.nlm.nih.gov \\
\hline Peptide & Mus musculus & Uniprot & 115,377 & http://www.uniprot.org \\
\hline
\end{tabular}




\begin{tabular}{|l|l|l|r|l|}
\hline BE & Organism & Database & BEID & URL \\
\hline Peptide & Rattus norvegicus & Ens_translation & 30,245 & http://www.ensembl.org \\
\hline Peptide & Rattus norvegicus & Vega_translation & 1,260 & http://vega.sanger.ac.uk \\
\hline Peptide & Rattus norvegicus & RefSeq_peptide & 68,777 & https://www.ncbi.nlm.nih.gov \\
\hline Peptide & Rattus norvegicus & Uniprot & 40,789 & http://www.uniprot.org \\
\hline Object & Homo sapiens & MetaBase_object & 24,727 & https://portal.genego.com \\
\hline Object & Homo sapiens & GO_function & 4,130 & http://amigo.geneontology.org \\
\hline Object & Mus musculus & MetaBase_object & 22,000 & https://portal.genego.com \\
\hline Object & Mus musculus & GO_function & 4,094 & http://amigo.geneontology.org \\
\hline Object & Rattus norvegicus & MetaBase_object & 18,648 & https://portal.genego.com \\
\hline Object & Rattus norvegicus & GO_function & 4,060 & http://amigo.geneontology.org \\
\hline
\end{tabular}

Table 2. Genomics platforms available in the BED UCB-Human database instance.

\begin{tabular}{|l|l|l|}
\hline Name & Description & BE \\
\hline GPL6101 & Illumina ratRef-12 v1.0 expression beadchip & Gene \\
\hline GPL6947 & Illumina HumanHT-12 V3.0 expression beadchip & Gene \\
\hline GPL10558 & Illumina HumanHT-12 V4.0 expression beadchip & Gene \\
\hline GPL1355 & [Rat230_2] Affymetrix Rat Genome 230 2.0 Array & Gene \\
\hline GPL1261 & [Mouse430_2] Affymetrix Mouse Genome 430 2.0 Array & Gene \\
\hline GPL96 & [HG-U133A] Affymetrix Human Genome U133A Array & Gene \\
\hline GPL13158 & [HT_HG-U133_Plus_PM] Affymetrix HT HG-U133+ PM Array Plate & Gene \\
\hline GPL571 & [HG-U133A_2] Affymetrix Human Genome U133A 2.0 Array & Gene \\
\hline GPL570 & [HG-U133_Plus_2] Affymetrix Human Genome U133 Plus 2.0 Array & Gene \\
\hline GPL6480 & Agilent-014850 Whole Human Genome Microarray 4x44K G4112F & Gene \\
\hline GPL6885 & Illumina MouseRef-8 v2.0 expression beadchip & Transcript \\
\hline
\end{tabular}

All BEID from a specific scope can be obtained with the getBeIds function. A specific BEID and its relationships with others can be graphically explored with the exploreBe function. The use and the results of these two functions are exemplified in Results. Also, the functions guessIdOrigin and checkBeIds are used to guess and to check the scope of any list of identifiers. This set of functions is completed by the getBeIdSymbols, getBeIdNames, getGeneDescription and getBeIdDescription functions which provide different ways to annotate identifiers taking advantage of information related to connected BEID. Other functions, searchId and getRelevantIds, are also provided to seek relevant identifiers for a specific BE. These functions are used by a shiny (Chang et al., 2017) gadget (findBe) providing an interactive dictionary of BEID which is also made available as an Rstudio add-in (Allaire et al., 2017; Cheng, 2016).

As described above, the BED data model has been built to fulfill molecular biology processes in order to ensure the biological relevance of identifier mappings. The is_expressed_as and is_translated_in relationships correspond to the transcription and translation processes respectively whereas codes_for is a fuzzy relationship allowing the mapping of genes on objects not necessarily corresponding to the same kind of biological molecules. These processes are described in different databases with different levels of granularity. For example, Ensembl (Zerbino et al., 2018) provides possible transcripts for each gene specifying which of them is canonical. The getDirectProduct and getDirectorigin functions allow the user to retrieve direct products or direct origins of such molecular biology processes. 
The automatic conversion of identifiers from one scope to another is handled by the convBeIds function. This conversion process can be applied directly on lists of identifiers (convBeIdLists) or on data frames with an identifier column (convDfBeIds). Because such conversion can be intricate, the exploreConvPath function is provided to display the shortest relevant paths between two identifiers. These functions are also exemplified in Results and Use case.

Converting thousands of identifiers can take some time (generally a few seconds). Also, such conversions are often recurrent and redundant. In order to improve the performance for such recurrent and redundant queries, a cache system has been implemented. The first time, the query is run on $\mathrm{Neo}^{\mathrm{j}}{ }^{\circledR}$ for all the relevant ID related to user input and the result is saved in a local file. Next time similar queries are requested, the system does not call $\mathrm{Neo} \mathrm{j}^{\circledast}$ but loads the cached results and filters it according to user input. By default, the cache is flushed when the system detects inconsistencies with the BED database. If needed, it can also be manually flushed by using the 1sBedCache and clearBedCache functions.

\section{Operation}

Minimal system requirements for running BED and neo2R R packages:

- $\mathbf{R} \geq 3.4$

- Operating system: Linux, macOS, Windows

- Memory $\geq 4$ GB RAM

The graph database has been implemented with $\mathrm{Neo}^{\circledR} \mathrm{j}^{\circledR}$ version 3 (Neo4j inc, 2017). The BED R package depends on the following packages available in the Comprehensive R Archive Network (CRAN):

- visNetwork (Almende et al., 2017)

- $\operatorname{dplyr}$ (Wickham et al., 2017)

- htmltools (RStudio inc, 2017)

- $D T$ (Xie, 2016)

- shiny (Chang et al., 2017)

- $\operatorname{miniUI}($ Cheng, 2016)

- rstudioapi (Allaire et al., 2017)

\section{Results}

The results below exemplify how BED efficiently tackle the three challenges described in the introduction. (1) The way identifiers are managed allow the mapping of deprecated identifiers. (2) The identifier conversion process takes advantage of the transitivity mechanism described in Methods to improve its completeness. (3) Mapping rules between different types of biological entities (BE) allow the correct and automatic conversion of identifiers. Finally, BED run time has been compared to three other tools in different contexts. These results were obtained using the instance of the BED database described in Methods and which is available in Docker Hub: https://hub.docker.com/r/patzaw/bed-ucb-human/ (tag 2018-04-30).

\section{Management of identifiers}

Identifiers (ID) in BED can identify a biological entity (BEID) or a probe (ProbeID). The getBeIds function returns all ID from a specific scope. As described in Methods a scope is defined by its type (the type of BE or "Probe"), its source (a database for BEID or a platform for ProbeID) and the organism to which it refers. For example, the following code returns all the Ensembl identifiers of human genes.

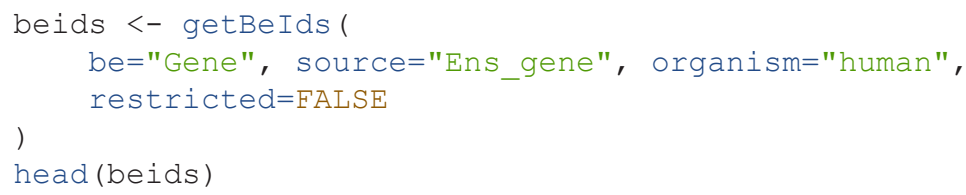




\begin{tabular}{|c|c|c|c|c|c|c|}
\hline \#\# & & id & preferred & Gene & db. version & $\mathrm{db}$. deprecated \\
\hline \#\# & 83452 & ENSG00000276626 & TRUE & 65397 & 92 & FALSE \\
\hline \#\# & 83453 & ENSG00000199595 & TRUE & 65401 & 92 & FALSE \\
\hline \#\# & 83454 & ENSG00000201381 & FALSE & 65401 & 54 & 20090519 \\
\hline \#\# & 83468 & ENSG00000200605 & TRUE & 65406 & 92 & FALSE \\
\hline \#\# & 83469 & ENSG00000206757 & FALSE & 65406 & 54 & 20090519 \\
\hline \#\# & 83455 & ENSG00000207395 & TRUE & 65407 & 92 & FALSE \\
\hline
\end{tabular}

The id column corresponds to the BEID from the source of interest. The column named according to the type of $\mathrm{BE}$ (in this case Gene) corresponds to the internal identifiers of the related BE. This internal identifier is not a stable reference that can be used as such. Nevertheless, it is useful to identify BEID identifying the same BE. In the example above, even if most Gene BE are identified by only one Ensembl gene BEID, many of them are identified by two or more $(6,031 / 59,901 \approx 10 \%) ; 277 \mathrm{BE}$ are even identified by more than 10 Ensembl BEID (Figure 2a). In this case, most of these redundancies come from deprecated BEID from former versions of the Ensembl database (version in use here: 92) and can be excluded by setting the restricted parameter to TRUE when calling the getBeIds function (Figure 2b). However, many BE are still identified by two or more current Ensembl BEID $(2,944 / 59,901 \approx 5 \%)$. This result comes from the way the BED database is constructed: When two BEID from the same resource correspond to the same BEID in another resource (correspond_to relationship in the data model), all these BEID are considered to identify the same BE.

A complex example of such mapping is shown in Figure 3 mapping all the BEID of the human TAS2R8 gene which codes for a protein of the family of candidate taste receptors. There are three identifiers corresponding to this gene symbol in Ensembl. All these three BEID correspond to the same Entrez gene and the same HGNC identifiers. All these BEID are thus considered to identify the same gene. It turns out that the three Ensembl BEID correspond to the same gene mapped on different sequence versions of the chromosome 12: the canonical (ENSG00000121314), CHR_HSCHR12_2_CTG2 (ENSG00000272712) and CHR_HSCHR12_3_CTG2 (ENSG00000277316). This information provided by Ensembl is encoded in the seq_region attribute for each Ensembl BEID (see data model) and is used to define preferred BEID which are mapped on canonical versions of chromosome sequences. The ENSG00000272712 identifier shows also a complex history in former Ensembl versions.

\section{Converting gene identifiers}

The main goal of BED is to convert identifiers from one scope to another easily, rapidly and with high completeness. It has been thought in order to allow recurring comparisons to each other of many lists of BEID from various origins.

(a)

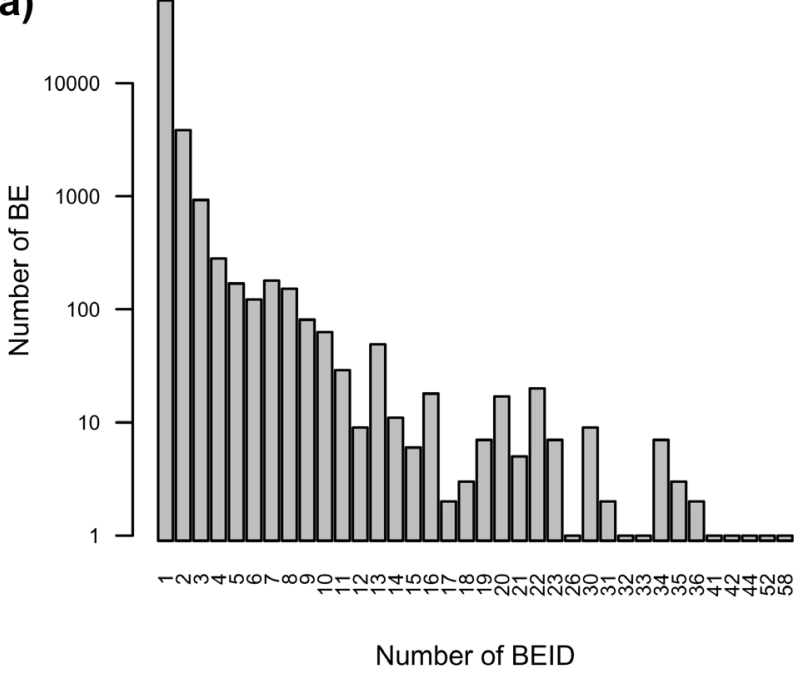

(b)

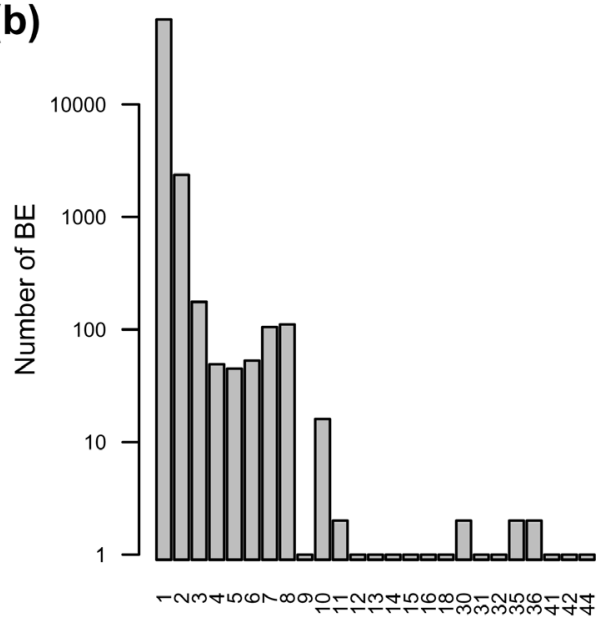

Number of BEID

Figure 2. Barplots showing the number (log scale) of gene BE identified by one or more Ensembl gene BEID. a) All Ensembl gene ID. b) Current Ensembl gene ID (version 92). 


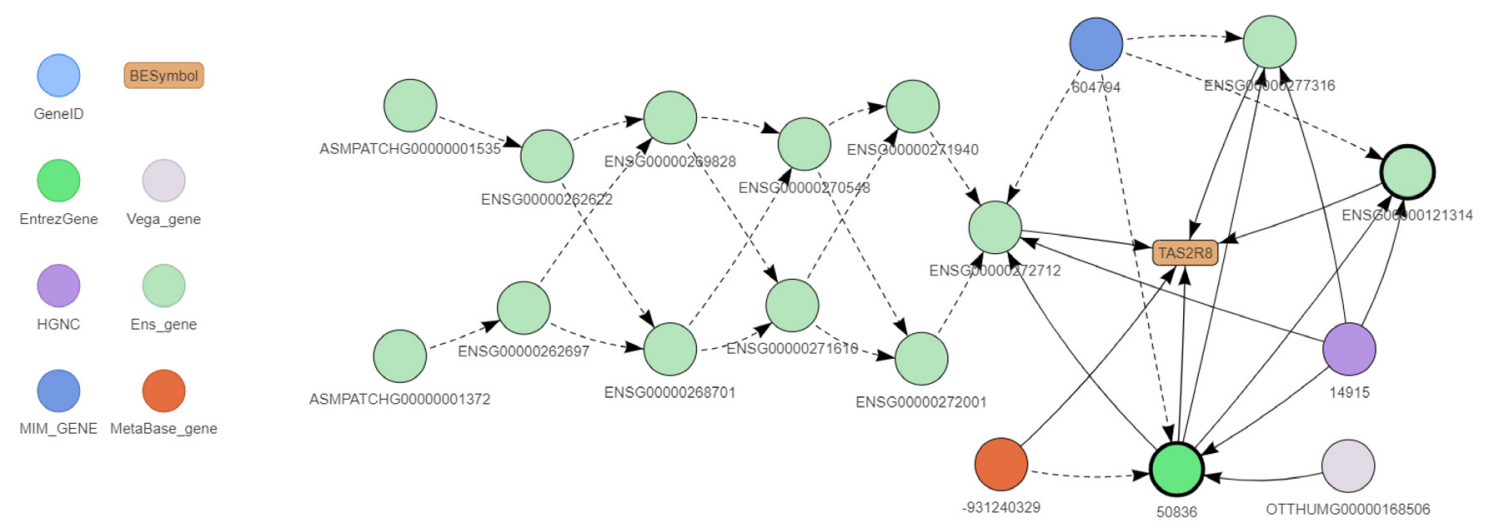

Figure 3. BED relationships between all the different identifiers of the human TAS2R8 gene recorded in the database. BEID are shown as circle and gene symbol in the rounded box. The color legend is shown to the left of the figure. BEID surrounded in bold correspond to preferred identifiers. Solid arrows represent correspond_to (between two nodes of identical shape) and is_known_as (between two nodes of different shapes) relationships. Dotted arrows represent is_replaced_by (between two nodes of identical color) and is_associated_to (between two nodes of different colors) relationships. This graph has been drawn with the exploreBe function.

A simple example regarding the conversion of human Ensembl gene to human Entrez gene identifiers is shown below and discussed hereafter. By setting the restricted parameter to TRUE the converted BEID are restricted to current - non-deprecated - version of Entrez gene identifiers. Nevertheless, all the input BEID are taken into account, current and deprecated ones.

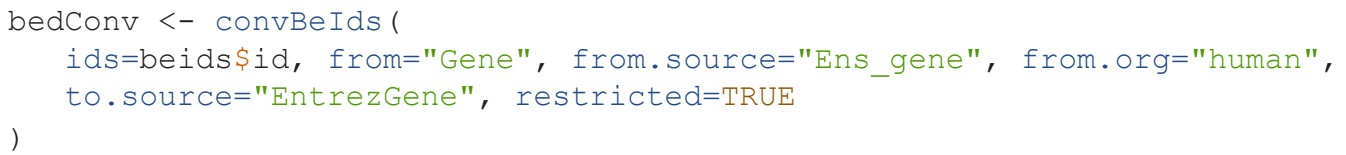

Among all the 69,056 human Ensembl gene identifiers available in the database, 22,056 (32\%) were not converted to any human Entrez gene identifier: 21,416 (33\%) of the 65,256 non-deprecated and 640 (17\%) of the 3,800 deprecated identifiers.

In order to assess the improvement of completeness achieved by BED, we compared it to three other tools: biomaRt (Durinck et al., 2009; Kinsella et al., 2011), mygene (Mark et al., 2014; Wu et al., 2013), and gProfileR (Reimand et al., 2016a; Reimand et al., 2016b). All these tools were used on May 03, 2018 to perform the same conversion task. At that time, biomaRt was based on the Ensembl 92 release (as the BED database instance), mygene on release 91 and gProfileR on release 90.

The numbers of human Ensembl gene identifiers successfully converted by each method are compared in Figure 4. Mappings returned only by gProfileR or by mygene $(33+94+76)$ are available in releases 90 and 91 of Ensembl respectively but not in release 92. They probably correspond to deprecated cross-references. Conversely, mappings returned by both BED and biomaRt but neither by gProfileR nor mygene (319) are available in release 92 of Ensembl but not in releases 91 or 90 . All the gene identifiers successfully converted by biomaRt were also converted by BED. However, BED was able to map at least 18,080 more identifiers than all the other tools (Figure 4a). A few of these mappings $(3,160)$ are explained by the fact that BED is the only tool mapping deprecated identifiers to current versions. Nevertheless, even when focusing on the mapping of current versions of Ensembl identifiers, BED was able to map 14,920 more identifiers than all the other tools (Figure 4b). A few of these mappings (683) are directly provided by the NCBI. But most of them (14,237) are inferred from a mapping of the Ensembl and Entrez gene identifiers to the same HGNC (Gray et al., 2015) identifier.

We assessed the validity of the mappings by comparing the location on the human genome (GRCh38) of Ensembl gene identifiers as reported by Ensembl and the location of the corresponding Entrez gene identifiers as reported by the NCBI. If the mapping between two identifiers is correct their location on the genome should 
(a)

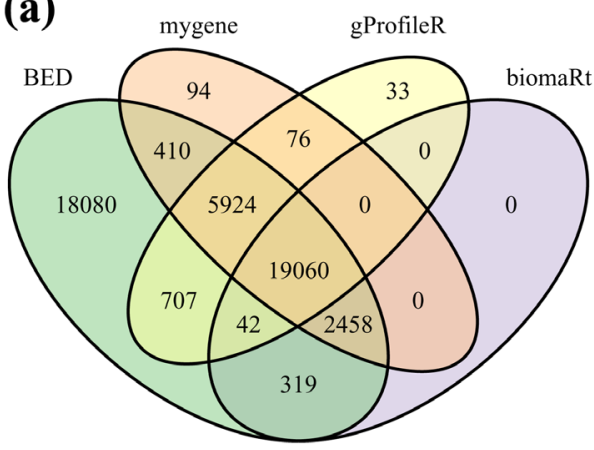

(b)

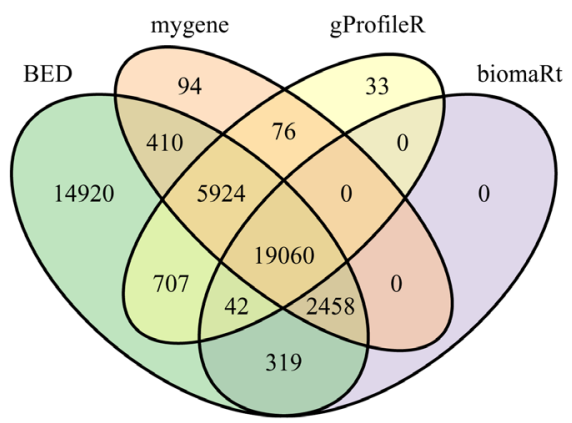

Figure 4. Venn diagrams showing the number of human Ensembl gene identifiers mapped to at least one human Entrez gene identifier by the different tested tools when focusing (a) on all 69,056 or (b) on current 65,256 BEID (Ensembl 92 release).

be identical or highly similar (only gene identifiers located on canonical versions of chromosome sequences were considered for this comparison). We compared the following mapping results:

- The "Reference" mappings provided by biomaRt for identifiers successfully converted by all the tools were considered as the reference (19,166 regard genes on canonical versions of chromosomes).

- The "Former" mappings provided by mygene for identifiers successfully converted by mygene but neither by biomaRt nor BED correspond to mappings only available in the former version (91) of Ensembl (67 regard genes on canonical versions of chromosomes).

- The "BED" mappings provided by BED for identifiers successfully converted by BED but not by any of the three other tools correspond to the mappings achieved mostly thanks to transitivity through HGNC identifiers (12,913 regard genes on canonical versions of chromosomes).

As shown in Figure 5a, the proportion of former cross-referenced identifiers ("Former") located on different chromosomes is much higher than for the reference ("Reference"). The proportion of BED cross-referenced identifiers ("BED") located on different chromosomes is higher than for "Reference" but still low. Figures 5.a and $\mathrm{b}$ show that inconsistencies in gene start location and in gene size are much more important in "Former" mappings than in "Reference" mappings. Such difference is not observed between the "Reference" mappings and the additional mappings provided by BED. These results show that the general quality of "Former" mappings is lower than the "Reference", which is in agreement with their deprecation. More interestingly, additional mappings provided by BED are of similar quality to the "Reference" making their use as valuable.

As shown above, additional mappings between Ensembl and Entrez gene identifiers inferred thanks to the use of HGNC cross-references by BED are many and of good quality. However, this transitivity mechanism is not always desirable depending on how different resources define biological entities. It is especially true for gene which is an unstable concept as described by Gerstein et al. (2007). For example, in Entrez the Hs.103110 UniGene identifier is mapped to 5465 and to 150383 Entrez gene identifiers which correspond to two different genes in Entrez (PPARA and CDPF1) but also in Ensembl (ENSG00000186951 and ENSG00000205643) and in HGNC (9232 and 33710). These two genes are located closely on the same chromosome but at different positions and on different directions. The same feature has been observed for many UniGene identifiers which makes UniGene unsuitable for transitivity mappings between gene identifiers. Therefore, in this instance of the BED database, as described in Methods, cross-references between Ensembl and Unigene gene identifiers are considered as is_associated_to relationships avoiding transitivity.

\section{Mapping rules}

Beside cross-referencing identifiers of identical type of BE, BED uses the biological relationship between genes, transcript and peptides to convert identifiers across different type of BE. For example, when converting peptides identifiers from the same species it uses only mapping done at the peptide level and does not use mapping to transcripts and genes. This strategy seems to be applied by biomaRt but not by mygene nor by 
(a)

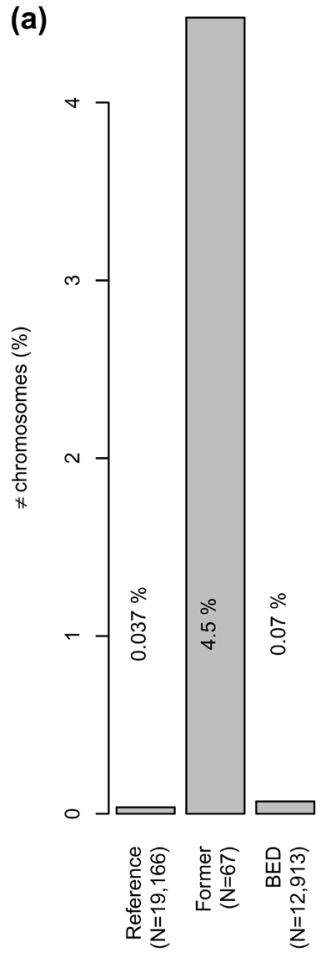

(b)

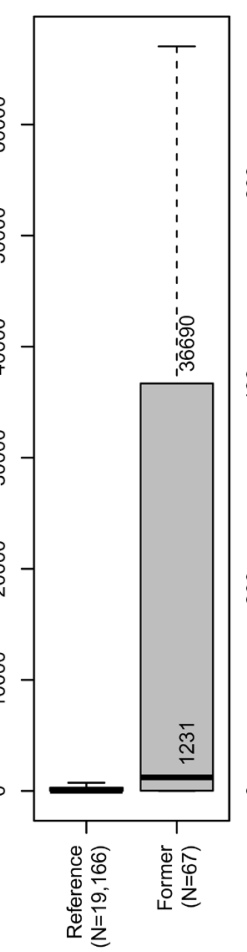

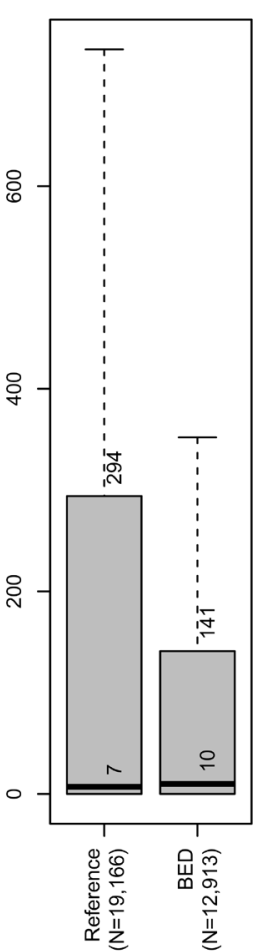
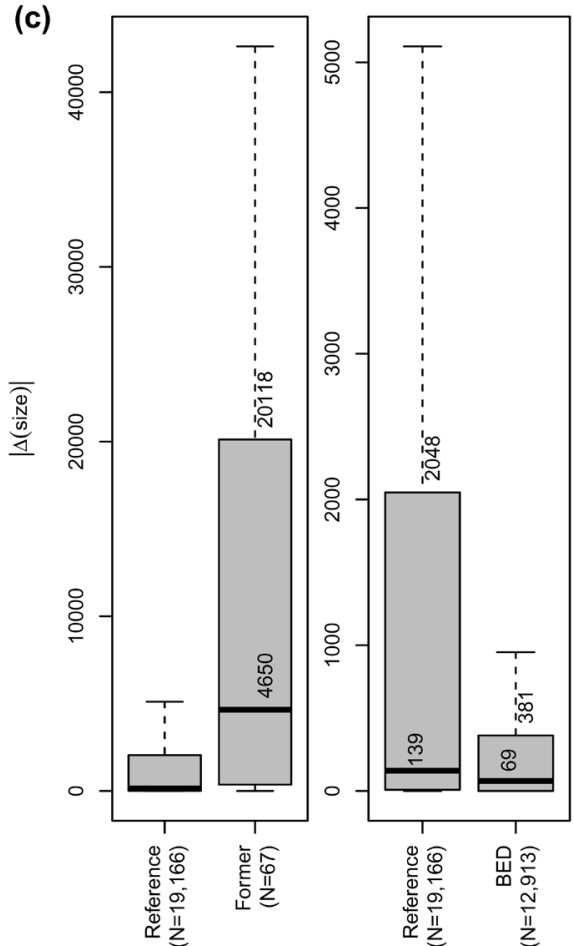

Figure 5. Comparison of gene locations provided by Ensembl for Ensembl identifiers and by the NCBI for the cross-referenced Entrez identifiers. "Reference" corresponds to mappings provided by biomaRt for identifiers successfully converted by all the tools. "Former" corresponds to mappings provided by mygene for identifiers successfully converted by mygene but neither by biomaRt nor BED. "BED" corresponds to mappings provided by BED for identifiers successfully converted by BED but not by any of the three other tools. Numbers of considered mappings $(\mathrm{N})$ in each group are indicated (see text for details). a) Proportion of cross-referenced identifiers located on different chromosomes. b) Absolute distance (base pairs) between gene start positions for cross-referenced identifiers located on the same chromosome. c) Absolute difference (base pairs) between gene sizes for crossreferenced identifiers located on the same chromosome. Median and third quartile values are indicated. Outliers are not shown. "BED" and "Former" results are compared to Reference on different boxplots because of scale shift.

gProfileR which map, for example, one Uniprot identifier to all the Ensembl peptide identifier coded by the same gene. For example, the A6NI28 Uniprot identifier is unambiguously mapped to the ENSP00000298815 Ensembl peptide identifier by BED and biomaRt but is wrongly mapped to three additional Ensembl peptide identifiers by mygene and gProfileR (ENSP00000431776, ENSP00000434304 and ENSP00000435961) which are encoded by the same gene (ENSG00000165895).

Furthermore, in biomaRt, mygene and gProfileR, mapping of BEIDs that are not genes from two different organisms using orthologs information requires at least two steps: one to find the ortholog genes and the other to find the relevant BEID. These two steps are integrated and transparent in BED.

In general, thanks to the BED data model and to mapping rules, identifier conversions from one scope to another are biologically relevant and automatic as exemplified in the use case described below.

Because all these indirect mappings can be intricate, BED provides a function to show the shortest relevant paths between two different identifiers (Figure 6).

\section{Performance}

The time taken by BED to convert different sets of identifiers from one scope to another has been compared to the time taken by biomaRt, mygene and gProfileR. Three different queries have been executed starting from 100 or 20,000 identifiers.

As shown in Figure 7, BED outperforms mygene and gProfileR in all the tested cases even when not using its cache system. It also outperforms biomaRt for converting Affymetrix probe ID into Ensembl mouse 

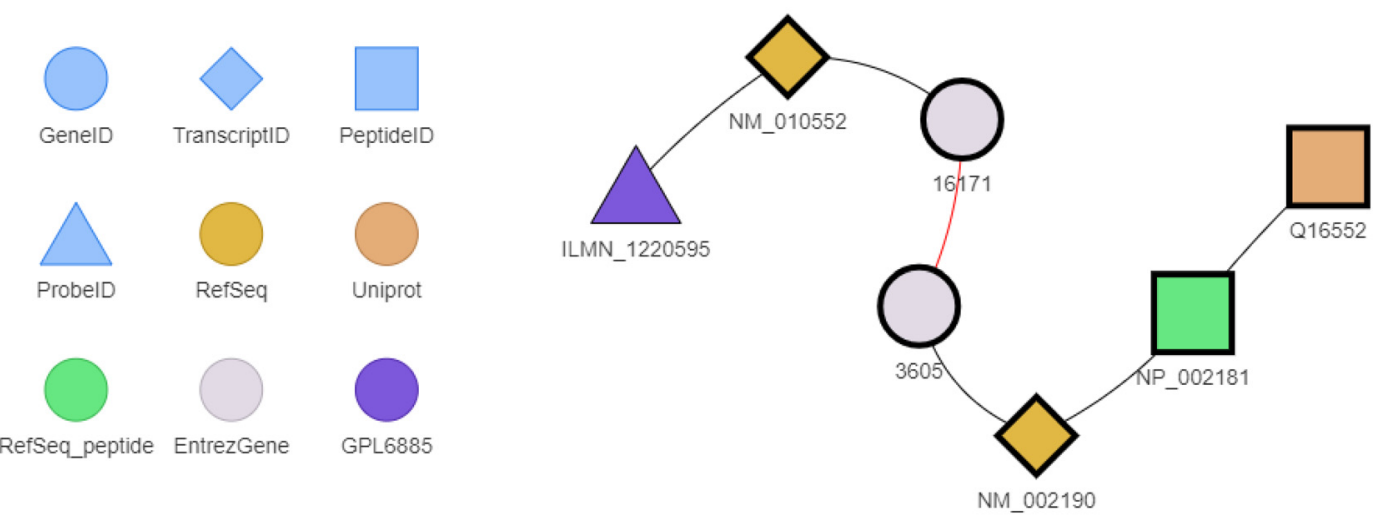

Figure 6. BED conversion shortest path between the ILMN_1220595 probe identifier targeting a transcript of the mouse II17a gene and the Uniprot Q16552 identifier of the human IL17 protein. The legend is shown to the left of the figure. The red edge represents the is_homolog_of relationship. BEID surrounded in bold correspond to preferred identifiers. This graph has been drawn with the exploreConvPath function.
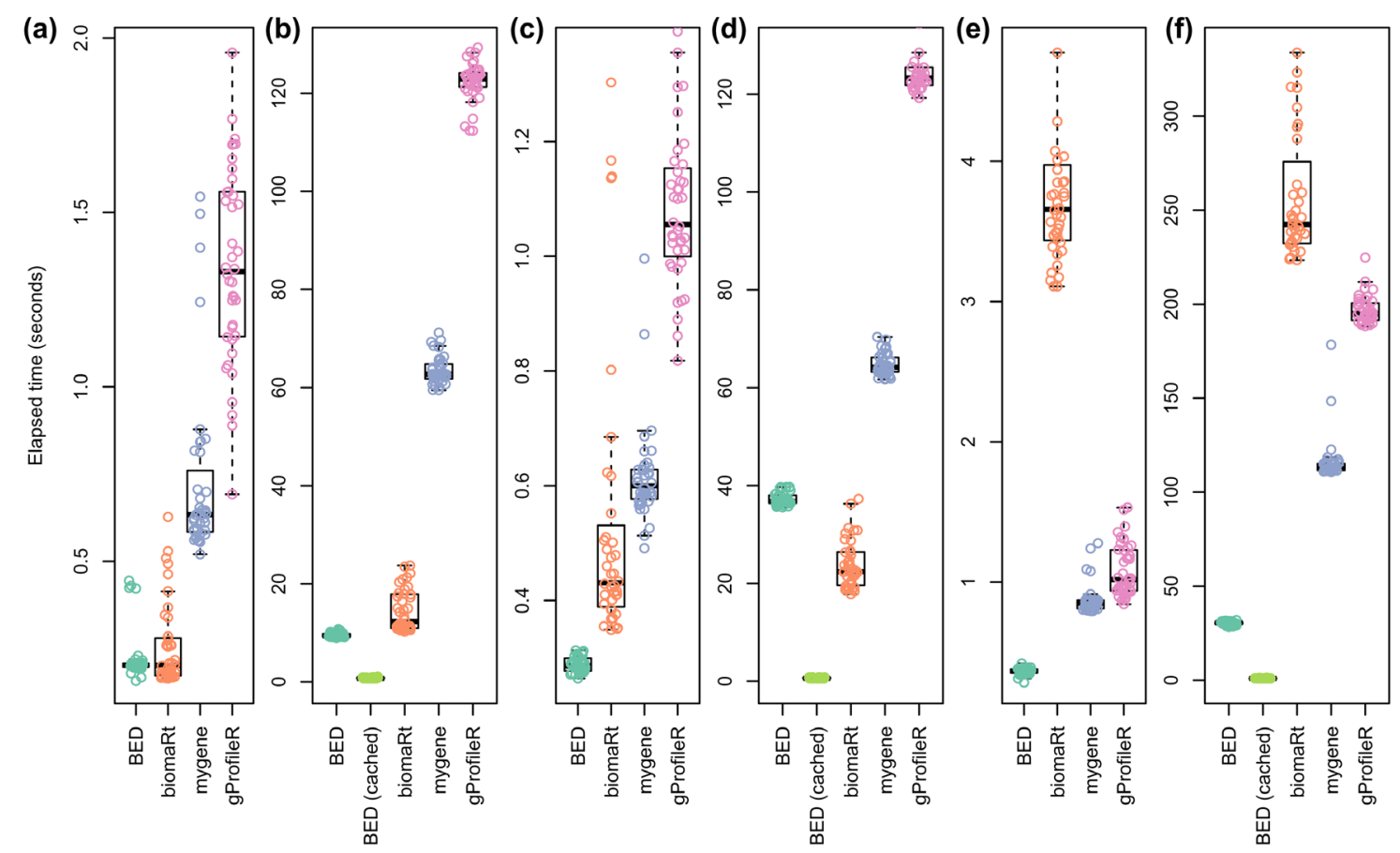

Figure 7. Comparison of the time taken to convert a set of randomly selected BE identifiers. Random selections of 100 or 20,000 identifiers have been executed 40 times before conversion by each considered tools. BED performance was assessed without using its cache system for all the queries and also using its cache system for queries regarding 20,000 identifiers. a) Conversion of 100 Ensembl human gene ID into Entrez gene ID. b) Conversion of 20,000 Ensembl human gene ID into Entrez gene ID. c) Conversion of 100 Uniprot mouse peptide ID into Ensembl transcript ID. d) Conversion of 20,000 Uniprot mouse peptide ID into Ensembl transcript ID. e) Conversion of 100 Affymetrix probe ID into Ensembl mouse peptide ID. f) Conversion of 20,000 Affymetrix probe ID into Ensembl mouse peptide ID.

peptide ID (Figures 7.e and 7.f). Without using its cache system, BED performs equally well as biomaRt for converting Ensembl human gene ID into Entrez gene ID (Figures 7.a and 7.b). It outperforms biomaRt for converting 100 Uniprot mouse peptide ID into Ensembl transcript ID (Figures 7.c) but is outperformed by biomaRt when 20,000 identifiers are considered (Figures 7.d). Nevertheless, when cache is used, BED outperforms all the tools whatever the conversion scenario (Figures 7.b, 7.d and 7.f). These results show that BED, as a dedicated and locally available tool, is a very efficient option to convert large lists of identifiers on the fly and recurrently. 
Use case: comparing transcriptomics data sets

In this use case, we show how BED facilitates the comparison of data sets relying on identifiers with different scopes. Indeed, the mechanisms implemented in the BED R package make easy the conversion of a set of identifiers from one scope to any other. Thus, the comparison of data from different genome wide experiments is straightforward from a technical point of view.

To exemplify this statement, we show below how to compare differential expression data from three experiments addressing the understanding of Psoriasis mechanisms with different experimental designs and platforms:

- Nair et al. (2009) compared gene expression data in lesional and non-lesional skin from Psoriasis patients using Affymetrix GeneChip Human Genome U133 Plus 2.0. Starting from published data (Array-Express (https://www.ebi.ac.uk/arrayexpress) accession number: E-GEOD-13355) we recomputed differential expression between these two groups of samples.

- Chiricozzi et al. (2011) measured transcriptomics responses to IL-17 and TNF- $\alpha$ cytokines in human keratinocytes using Illumina HumanHT-12 v3.0 Expression BeadChips. Starting from published data (E-GEOD-24767) we recomputed differential expression between keratinocytes co-stimulated with both cytokines and controls.

- Swindell et al. (2011) compared gene expression data in lesional and non-lesional skin from five psoriasis mouse models using Affymetrix GeneChip Mouse Genome 430 2.0. Starting from published data (E-GEOD-27628) we recomputed differential expression between affected and non-affected skin samples.

The first rows of these three data tables are shown below.

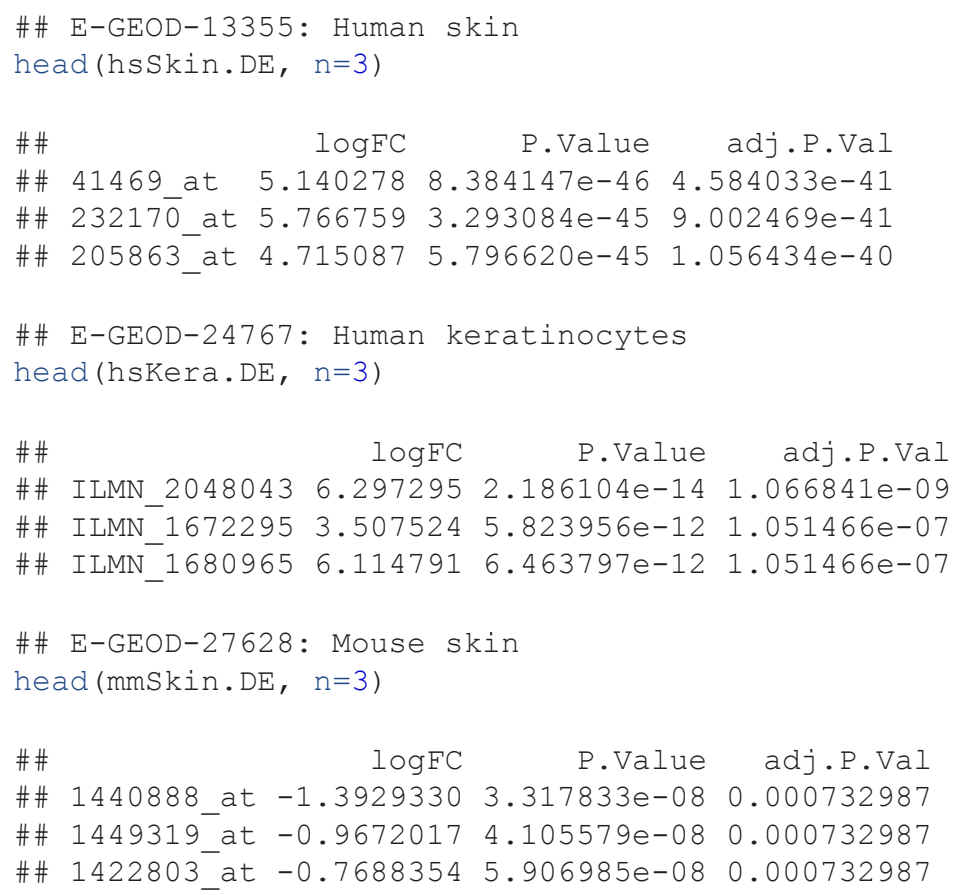

The aim of the following commands is to allow the comparison of the three logFC values by converting ProbeID (row names) of one or the other data set. The function guessIdorigin is used to identify the scope of the different sets of identifiers.

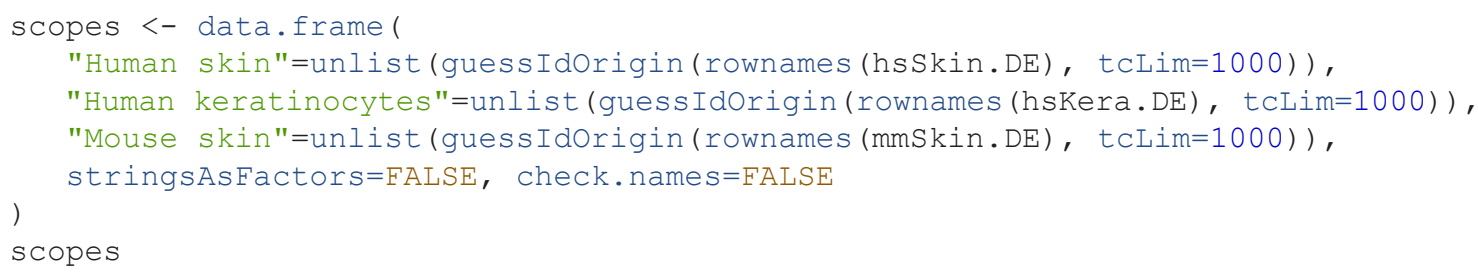




$\begin{array}{lrrr}\# \# & \text { Human skin Human keratinocytes } & \text { Mouse skin } \\ \# \# \text { be } & \text { Probe } & \text { Probe } & \text { Probe } \\ \# \# \text { source } & \text { GPL570 } & \text { GPL10558 } & \text { GPLI261 } \\ \text { \#\# organism Homo sapiens } & \text { Homo sapiens Mus musculus }\end{array}$

To compare the two human data sets, the keratinocyte data set can be converted to the same scope of the skin data set using the convDfBeIds function. After conversion, the two data sets can be merged before computing the correlation between $\operatorname{logFC}$ values.

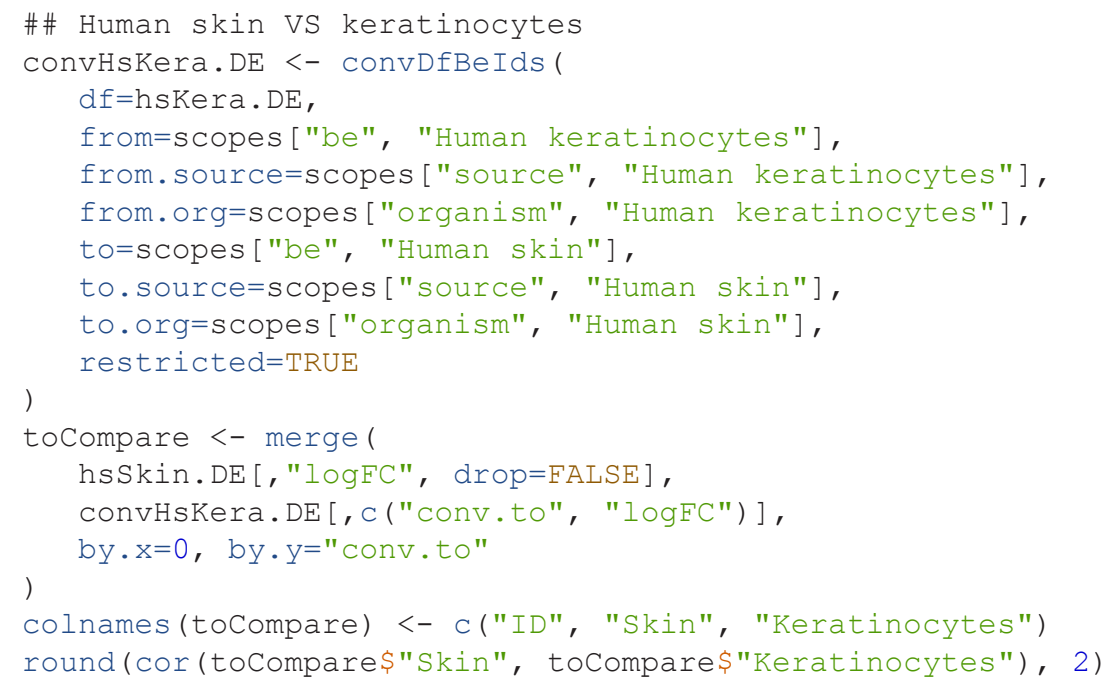

A similar procedure can be applied to compare the two skin data sets or to compare mouse skin and human keratinocytes.

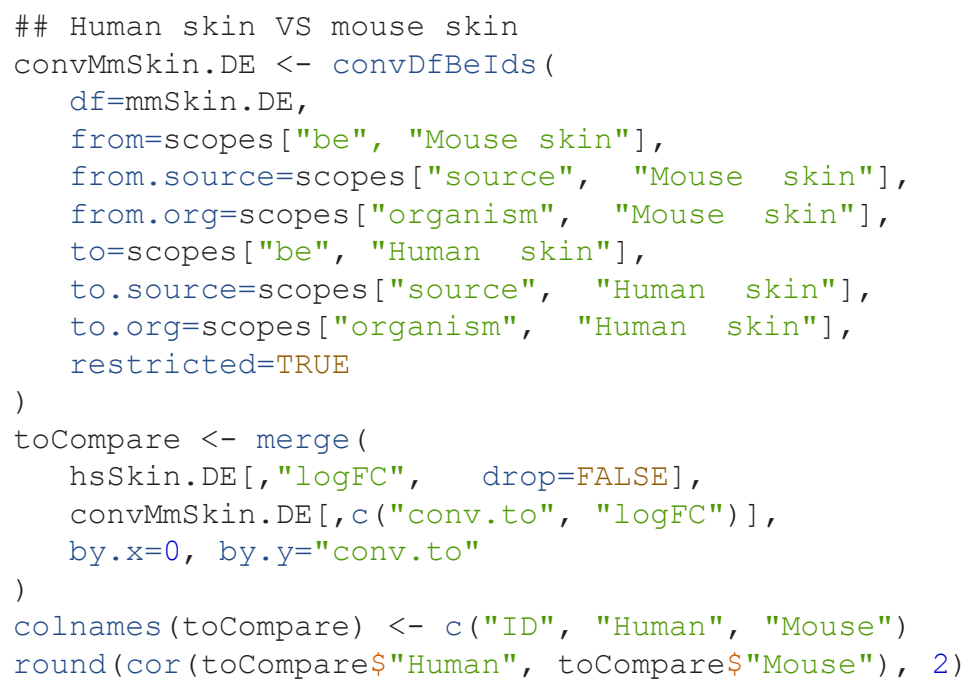




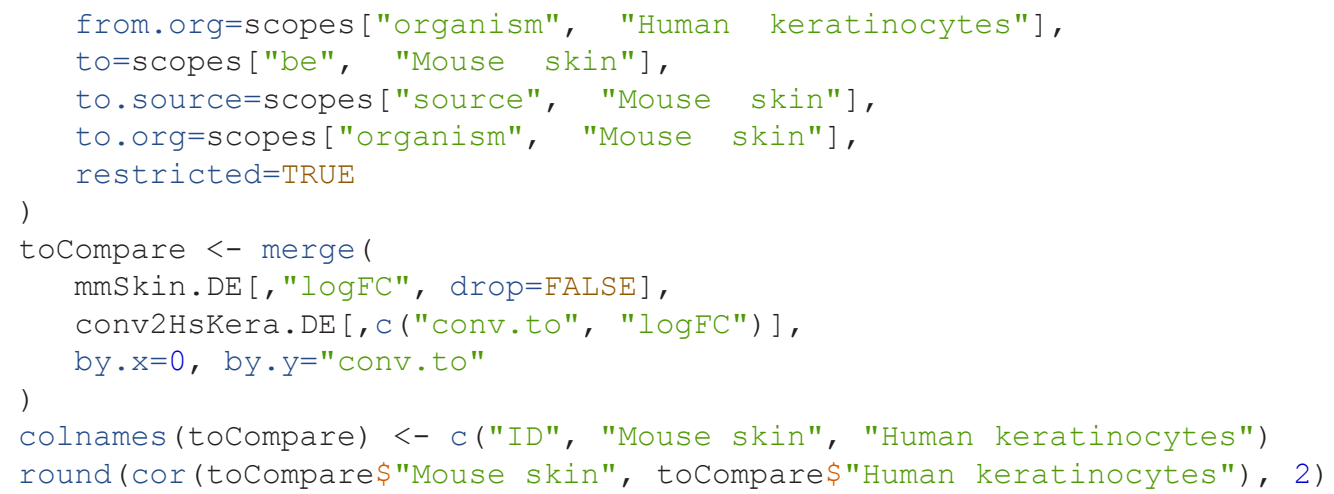

As shown above, converting the identifier scope of transcriptomic data sets is straightforward and quickly executed using BED. It makes the comparison (such as correlation analyses performed in this use case) and the integration of multiple heterogeneous data very easy to achieve.

Additional examples of BED functionalities are provided in the R package vignette.

\section{Conclusions}

The appearance of "omics" technologies, biological knowledge databases and systems biology analytical approaches have opened the possibility to integrate various data sets to get a better understanding of biological processes underlying different complex phenotypes such as diseases. However, this promising interoperability of data sets is largely hampered by the heterogeneity of identifiers used by technical platforms but also those used by knowledge databases to organize information. Comparing and integrating all these data requires the ability to map the identifiers on which each resource relies. Many tools have been developed to achieve this task (e.g. Kinsella et al. (2011); Reimand et al. (2016a); Wu et al. (2013) or van Iersel et al. (2010)). However, we identified three challenges generally not addressed by the available tools:

- Information provided by different data sources is not leveraged to increase the completeness of identifier conversion.

- Deprecated identifiers, used in former versions of resources, are not available anymore for conversion in up-to-date versions of mapping tools, damaging the integration of historical data sets.

- The mapping between very different scopes of identifiers is either difficult to automate or not biologically relevant.

BED is a system dedicated to the mapping between identifiers of molecular biological entities. It relies on a graph data model implemented with $\mathrm{Neo} \mathrm{j}^{\circledR}$ and on rules coded in an $\mathrm{R}$ package. BED leverages mapping information provided by different resources in order to increase the mapping completeness between each of them. It also allows the mapping of deprecated identifiers. Rules are used to automatically convert identifiers from one scope to another using the most appropriate path.

The intent of BED is to be tailored to specific needs and beside functions for querying the system the BED $\mathrm{R}$ package provides functions to build custom instances of the database, including internal or proprietary resources for example. Database instances can be locally installed or shared across a community. This design combined with a cache system makes BED efficient for converting large lists of identifiers from and to a large variety of scopes. Because of our research field we provide an instance focused on human, mouse and rat organisms. This database instance can be directly used in relevant projects but it can also be enriched depending on user or community needs.

Beside a casual use for analyzing and comparing data in the frame of a research project, BED can be advantageously employed by systems dealing with biological and molecular information from heterogeneous sources. Indeed, various pieces of knowledge can be efficiently managed in their original scope (e.g. gene ID for genomics 
or epigenomics data, transcript ID for transcriptomics data and protein ID for proteomics data). Thanks to the automatic and fast conversion of identifiers all these data can then be compared to each other or to any user input. We do not provide BED API for other languages than R but we expect that publishing the source code under an open source license (GPL-3) will encourage other developers to extend or improve it according to their needs and their expertise.

\section{Software availability}

Latest source code is available at:

https://github.com/patzaw/BED

https://github.com/patzaw/neo2R

Archived source code as at time of publication:

http://doi.org/10.5281/zenodo.1244150 (Godard, 2018a)

http://doi.org/10.5281/zenodo.1167670 (Godard, 2018b)

Archived database docker image as at time of publication:

http://doi.org/10.5281/zenodo.1311362 (Godard, 2018c)

Software is available to use under a GPL-3 license

Competing interests

No competing interests were disclosed.

Grant information

This work was entirely supported by UCB Pharma. The authors declared that no grants were involved in supporting this work.

Acknowledgments

We are grateful to Frédéric Vanclef, Malte Lucken, Liesbeth François, Matthew Page, Massimo de Francesco, and Marina Bessarabova for fruitful discussions and constructive criticisms.

Allaire JJ, Wickham H, Ushey K, et al.: rstudioapi: Safely Access the RStudio API. 2017.

Reference Source

Almende BV, Thieurmel B, Robert T: visNetwork: Network

Visualization using 'vis.js' Library. 2017.

Reference Source

Chang W, Cheng J, Allaire JJ, et al:: shiny: Web Application

Framework for R. 2017.

Reference Source

Cheng J: miniUl: Shiny UI Widgets for Small Screens. 2016.

Reference Source

Chiricozzi A, Guttman-Yassky E, Suárez-Fariñas M, et al.: Integrative responses to IL-17 and TNF- $\alpha$ in human keratinocytes account

for key inflammatory pathogenic circuits in psoriasis. J Invest

Dermatol. 2011; 131(3): 677-87.

PubMed Abstract | Publisher Full Text

Clarivate Analytics: MetaCore delivers high-quality biological systems content in context. 2017.

Reference Source

CRAN: The Comprehensive R Archive Network.

Reference Source

Crick F: Central dogma of molecular biology. Nature. 1970; 227(5258): 561-563.

PubMed Abstract | Publisher Full Text

Dai X, Li J, Liu T, et al:: HRGRN: A Graph Search-Empowered

Integrative Database of Arabidopsis Signaling Transduction,

Metabolism and Gene Regulation Networks. Plant Cell Physiol.

2016; 57(1): e12.

PubMed Abstract | Publisher Full Text | Free Full Text
Davis S, Meltzer PS: GEOquery: a bridge between the Gene Expression Omnibus (GEO) and BioConductor. Bioinformatics. 2007; 23(14): 1846-1847.

PubMed Abstract | Publisher Full Text

Docker inc: Docker Community Edition. 2017

Reference Source

Durinck S, Spellman PT, Birney E, et al:: Mapping identifiers for the integration of genomic datasets with the R/Bioconductor package biomaRt. Nat Protoc. 2009; 4(8): 1184-1191.

PubMed Abstract | Publisher Full Text | Free Full Text

Fabregat A, Korninger F, Viteri $G$, et al.: Reactome graph database: Efficient access to complex pathway data. PLOS Comput Biol. 2018; 14(1): e1005968.

PubMed Abstract | Publisher Full Text | Free Full Text

Gerstein MB, Bruce C, Rozowsky JS, et al:: What is a gene, post-

ENCODE? History and updated definition. Genome Res. 2007;

17(6): 669-81.

PubMed Abstract | Publisher Full Text

Godard P: patzaw/BED: Publication (V2) release (Version v1.1.0).

Zenodo. 2018a.

Data Source

Godard P: patzaw/neo2R: Publication release (Version v1.0.0).

Zenodo. 2018b.

Data Source

Godard P: docker-bed-ucb-human-2018.04.30 (Version 2018.04.30).

Zenodo. 2018c

Data Source

Gray KA, Yates B, Seal RL, et al:: Genenames.org: the HGNC

resources in 2015. Nucleic Acids Res. 2015; 43(Database issue): 
D1079-1085.

PubMed Abstract | Publisher Full Text | Free Full Text

Kinsella RJ, Kähäri A, Haider S, et al.: Ensembl BioMarts: a hub for data retrieval across taxonomic space. Database (Oxford). 2011;

2011: bar030.

PubMed Abstract | Publisher Full Text | Free Full Text

Mark A, Thompson R, Afrasiabi C, et al.: mygene: Access MyGene.

Info services. 2014

Publisher Full Text

Nair RP, Duffin KC, Helms C, et al.: Genome-wide scan reveals association of psoriasis with IL-23 and NF-kappaB pathways. Nat Genet. 2009; 41(2): 199-204.

PubMed Abstract | Publisher Full Text | Free Full Text

NCBI Resource Coordinators: Database Resources of the Nationa Center for Biotechnology Information. Nucleic Acids Res. 2017; 45(D1): D12-D17.

PubMed Abstract | Publisher Full Text | Free Full Text

Neo4j inc: Neo4j Community Edition. 2017.

Reference Source

Pareja-Tobes $\mathrm{P}$, Tobes R, Manrique M, et al.: Bio4j: a highperformance cloud-enabled graph-based data platform. bioRxiv. 2015; 016758.

Publisher Full Text

Reimand J, Arak T, Adler P, et al.: g:Profiler-a web server for functional interpretation of gene lists (2016 update). Nucleic Acids Res. 2016a; 44(W1): W83-89.

PubMed Abstract | Publisher Full Text | Free Full Text

Reimand J, Kolde R, Arak T: gProfileR: Interface to the 'g:Profiler' Toolkit. 2016b.

Reference Source

R Core Team: R: A Language and Environment for Statistical

Computing. R Foundation for Statistical Computing, Vienna, Austria;
2017.

Reference Source

RStudio inc: htmltools: Tools for HTML. 2017.

Reference Source

Swindell WR, Johnston A, Carbajal S, et al:: Genome-wide expression profiling of five mouse models identifies similarities and differences with human psoriasis. PLoS One. 2011; 6(4): e18266.

PubMed Abstract | Publisher Full Text | Free Full Text

The UniProt Consortium: UniProt: the universal protein knowledgebase. Nucleic Acids Res. 2017; 45(D1): D158-D169. PubMed Abstract | Publisher Full Text | Free Full Text

van lersel MP, Pico AR, Kelder T, et al.: The BridgeDb framework: standardized access to gene, protein and metabolite identifier

mapping services. BMC Bioinformatics. 2010; 11: 5 .

PubMed Abstract | Publisher Full Text | Free Full Tex

Wickham H, Francois R, Henry L, et al.: dplyr: A Grammar of Data Manipulation. 2017.

Reference Source

Wu C, Macleod I, Su Al: BioGPS and MyGene.info: organizing online, gene-centric information. Nucleic Acids Res. 2013

41(Database issue): D561-565.

PubMed Abstract | Publisher Full Text | Free Full Text

Xie Y: DT: A Wrapper of the JavaScript Library 'DataTables'. 2016. Reference Source

Yoon BH, Kim SK, Kim SY: Use of Graph Database for the Integration of Heterogeneous Biological Data. Genomics Inform. 2017; 15(1): 19-27.

PubMed Abstract | Publisher Full Text | Free Full Tex

Zerbino DR, Achuthan P, Akanni W, et al: Ensembl 2018. Nucleic

Acids Res. 2018; 46(D1): D754-D761.

PubMed Abstract | Publisher Full Text | Free Full Tex 


\section{Open Peer Review}

\section{Current Peer Review Status:}

\section{Version 3}

Reviewer Report 20 July 2018

https://doi.org/10.5256/f1000research.17046.r36266

(C) 2018 Simpson T. This is an open access peer review report distributed under the terms of the Creative Commons Attribution License, which permits unrestricted use, distribution, and reproduction in any medium, provided the original work is properly cited.

\section{T. Ian Simpson}

School of Informatics, University of Edinburgh, Edinburgh, UK, UK

Happy with these additional minor changes.

Competing Interests: No competing interests were disclosed.

Reviewer Expertise: Biological informatics, computational biology, neuroscience, statistics, machine learning

I confirm that I have read this submission and believe that I have an appropriate level of expertise to confirm that it is of an acceptable scientific standard.

\section{Version 2}

Reviewer Report 03 July 2018

https://doi.org/10.5256/f1000research.16282.r34080

(C) 2018 Summer-Kutmon $\mathbf{M}$ et al. This is an open access peer review report distributed under the terms of the Creative Commons Attribution License, which permits unrestricted use, distribution, and reproduction in any medium, provided the original work is properly cited.

\section{Denise Slenter}

Department of Bioinformatics - BiGCaT, Maastricht University, Maastricht, The Netherlands

\section{Martina M. Summer-Kutmon}

Department of Bioinformatics - BiGCaT, Maastricht University, Maastricht, The Netherlands

We would like to thank the authors for addressing our comments and incorporating them nicely in 
the new manuscript version. Additionally, they also added a lot more details and figures to many of the sections, which further clarify the approach and relevance of BED. We also really appreciate the added results and use case!

We only have some small remarks that might be nice to address:

Methods / Data model $\rightarrow$ deprecated identifiers are one of the challenges addressed by BED, but it is not discussed how those are represented in the data model.

Methods / Available database instance $\rightarrow$ it would be nice to provide a DOI for the specific docker instance release.

Results / Figure $6 \rightarrow$ legend should now say "The red edge" instead of "The red arrow".

Results / Figure $7 \rightarrow$ we believe legend for figure f should be "Conversion of 20,000

Affymetrix probe ID into Ensembl mouse peptide ID"

Competing Interests: We would like to note that the in the article mentioned BridgeDb framework is developed within our group.

We confirm that we have read this submission and believe that we have an appropriate level of expertise to confirm that it is of an acceptable scientific standard.

Reviewer Report 13 June 2018

https://doi.org/10.5256/f1000research.16282.r34079

(c) 2018 Simpson T. This is an open access peer review report distributed under the terms of the Creative Commons Attribution License, which permits unrestricted use, distribution, and reproduction in any medium, provided the original work is properly cited.

\section{T. Ian Simpson}

School of Informatics, University of Edinburgh, Edinburgh, UK, UK

Many thanks to the authors for making changes to the manuscript in response to my and the other reviewers comments. It is particularly nice to see the data for validation (Fig.5) and speed of execution (Fig.7) added. I am happy to recommend the article for indexing in this amended form.

Competing Interests: No competing interests were disclosed.

Reviewer Expertise: Biological informatics, computational biology, neuroscience, statistics, machine learning

I confirm that I have read this submission and believe that I have an appropriate level of expertise to confirm that it is of an acceptable scientific standard.

\section{Version 1}


Reviewer Report 26 March 2018

https://doi.org/10.5256/f1000research.15138.r31928

(c) 2018 Simpson T. This is an open access peer review report distributed under the terms of the Creative Commons Attribution License, which permits unrestricted use, distribution, and reproduction in any medium, provided the original work is properly cited.

\section{T. Ian Simpson}

School of Informatics, University of Edinburgh, Edinburgh, UK, UK

In this article the authors present BED, a biological entity database implemented as a Neo4J labelled property graph. In addition, they provide two R-packages (BED \& neo4J) for the construction and query of such graphs that adhere to their data model. These packages include utility functions to facilitate graph construction from a range of commonly used and publicly available data sources. The software and database are well documented and available through GitHub and Docker (as a Docker image) respectively and proved straight forward to install and run.

There are several elements of the current manuscript that warrant commentary:

1. Motivation/Rationale. The authors have correctly identified an important problem with the integration of biological data that has been addressed before, not least by the resources/tools mentioned in the manuscript (Biomart, my gene, g:Profiler amongst others). They have chosen a particularly good approach (labelled property graphs) to build the data architecture to address such a problem and one that has recently been used to great effect by the EMBL-EBI Reactome team to model data related to biological pathways. Currently this manuscript somewhat undersells the potential for the tools that have been developed. Whilst allusion is made at various points to the fact that the software developed could be used by others to develop custom resources very little is presented as to the suitability of their approach for such. The "Abstract" states that existing resources "cannot be customised and optimised for any specific use" which is not correct and should be removed or re-worded to clarify the author's meaning. Whilst the implementation presented here is focussed primarily on gene level mappings it should be made clear throughout the manuscript that the general approach used could be (and indeed has been, see citation) used in other really quite different biological data modelling scenarios.

2. Introduction. The issue of "transitivity" is raised here, this is a complex issue for many biological data types that are far removed from the rigid structures of ontologies that commonly enforce it by definition. The meaning of "transitivity" in the context used here is not clear and warrants further explanation. This is particularly important later in the article where decisions are being made about inferring mappings where they don't exist in the data. Some such inferences are entirely logical (e.g. using HGNC ids two link gene_ids between two resources that don't map directly to each other) but others are far more complex (e.g. mapping between species). The inclusion of deprecated identifiers is excellent and will help to close a notable gap in many existing resources for which mapping older data into more recent datasets can be extremely time consuming and frustrating. The authors comment on "mapping between different scopes" is unclear and should be 
clarified.

3. Methods. The sections "Feeding the Database" and "Querying the Database" are very brief and would benefit from much more detail about the functionality of the database creation and query system. Whilst these are covered in detail in the various pieces of documentation (including some very nice working examples) there is not enough in the manuscript itself to allow the reader to assess the available functionality.

\section{Use Cases.}

There appears to be a discrepancy in gene counts from the Ensembl examples used in this section; the first example calls human Ensembl genes and returns 59,515 genes the second states the total number of human Ensembl genes to be 68,460 .

Figure 3. illustrates a relationship graph including deprecated BEIDs. Whilst is_replaced_by is clear, it is not clear (or defined anywhere) what the meaning of is_associated_to is and how that differs from corresponds_to. This should be clarified in the text.

The sentence "The function guessIdOrigin..." appears out of place, unconnected to the surrounding text.

The statement "Five identifiers were only..." and the following sentence should be combined and re-worded so that the explanation as to why 5 BEIDs were uniquely found by gProfiler is clearer.

No validation or commentary has been presented to test the efficacy of inferences made by the query system. I would like to have seen an attempt made to check the veracity of mappings made in this way especially when the majority (c.80\%) of extra Ensmembl$>$ EntrezID mappings recovered via BED were inferred.

A "rough approximation" of timings for queries within BED and across other systems is not particularly informative. It would have been straightforward to automate a sampling approach to generate a mean response time (and a variance) to a defined set of query sizes/complexities to give the user a better understanding of how variable these response times are between the systems in practice. In addition, it would have been nice to see some analysis/discussion about the "scalability" of the system as this is likely to be of particular interest to end-users considering a similar modelling approach in other domains.

Figure5. The meaning of directionality here is not clear. Whilst I can see the benefit for provenance reasons i.e. a mapping from EntrezGene to RefSeq it's meaning here is somewhat moot.

The work presented in this manuscript promises to be very useful for researchers wanting to use LPGs for data integration. The implementation and deployment have been very well executed so that they can be readily adopted and modified by end-users.

\section{References}

1. Fabregat A, Jupe S, Matthews L, Sidiropoulos K, et al.: The Reactome Pathway Knowledgebase. Nucleic Acids Res. 2018; 46 (D1): D649-D655 PubMed Abstract | Publisher Full Text

Is the rationale for developing the new software tool clearly explained? Partly

Is the description of the software tool technically sound? 
Yes

Are sufficient details of the code, methods and analysis (if applicable) provided to allow replication of the software development and its use by others?

Yes

Is sufficient information provided to allow interpretation of the expected output datasets and any results generated using the tool?

Partly

Are the conclusions about the tool and its performance adequately supported by the findings presented in the article?

Partly

Competing Interests: No competing interests were disclosed.

Reviewer Expertise: Biological informatics, computational biology, neuroscience, statistics, machine learning

I confirm that I have read this submission and believe that I have an appropriate level of expertise to confirm that it is of an acceptable scientific standard, however I have significant reservations, as outlined above.

Author Response 26 Apr 2018

Patrice Godard, UCB, Belgium

Thanks for having taken the time to review this article and for your constructive comments that will help us to improve its quality. We are working on a second version. In the mean time we would like to provide you some feedback about the different issues you arose and how we are going to take your comments into account in the second version of our manuscript. Also we would like to inform you that we are going to use an updated version of the BED instance based on version 92 of Ensembl (released in April). Thus numbers provided in the article will slightly change in the next version.

Motivation/Rationale. The authors have correctly identified an important problem with the integration of biological data that has been addressed before, not least by the resources/tools mentioned in the manuscript (Biomart, my gene, g:Profiler amongst others). They have chosen a particularly good approach (labelled property graphs) to build the data architecture to address such a problem and one that has recently been used to great effect by the EMBLEBI Reactome team to model data related to biological pathways. Currently this manuscript somewhat undersells the potential for the tools that have been developed. Whilst allusion is made at various points to the fact that the software developed could be used by others to develop custom resources very little is presented as to the suitability of their approach for such.

We will address this point in the conclusion of the next version of the article by mentioning in which context BED can be used. 
The "Abstract" states that existing resources "cannot be customised and optimised for any specific use" which is not correct and should be removed or re-worded to clarify the author's meaning.

This statement has also been questioned by the other referee although slightly differently. We wanted to highlight the point that the way the mapping is done by most of these resources (excepted BridgeDB) cannot be customized, optimized or extended by the user according to his knowledge or to internal, non-public or non-standard information. These tools are dedicated to a particular domain: they are focused on species, type of identifiers, and update frequencies (as stated in BridgeDB publication by van Iersel et al. (2010)). It's convenient because ready to use but not flexible as BridgeDB or BED which allow an empowered user to focus on required information. We are going to modify this statement to make it less ambiguous in the next version.

Whilst the implementation presented here is focused primarily on gene level mappings it should be made clear throughout the manuscript that the general approach used could be (and indeed has been, see citation) used in other really quite different biological data modelling scenarios.

We will mention in the introduction that graph databases, specially Neo4j, have been used to model different kind of biological data.

Introduction. The issue of "transitivity" is raised here, this is a complex issue for many biological data types that are far removed from the rigid structures of ontologies that commonly enforce it by definition. The meaning of "transitivity" in the context used here is not clear and warrants further explanation. This is particularly important later in the article where decisions are being made about inferring mappings where they don't exist in the data. Some such inferences are entirely logical (e.g. using HGNC ids two link gene_ids between two resources that don't map directly to each other) but others are far more complex (e.g. mapping between species). The inclusion of deprecated identifiers is excellent and will help to close a notable gap in many existing resources for which mapping older data into more recent datasets can be extremely time consuming and frustrating. The authors comment on "mapping between different scopes" is unclear and should be clarified.

The transitivity mechanism is managed by the 2 following relationships: "corresponds_to" and "is_associated_to". On one hand the "corresponds_to" relationships make the mapping transitive since 2 BEIDs which are connected through this kind of relationship are considered to identify the same BE through an "identifies" relationship. On the other hand a BEID which "is_associated_to" to another one does not automatically "identify" the same BE making this kind of relationship not available for indirect mappings. When the BED database is fed, the user chooses which relationship should be of type "corresponds_to" or of type "is_associated_to". For example, in the instance we provide, cross references provided by Ensembl from Ensembl gene ID to Entrez, HGNC and Vega gene ID are considered as "corresponds_to" relationships whereas cross references to miRbase, Unigene and OMIM are considered as "is_associated_to" relationship. In Ensembl the Hs.745351 Unigene ID is mapped to ENSG00000184033 and to ENSG00000268651 Ensembl gene IDs which correspond to 2 different genes in Ensembl but also in Entrez and in HGNC and these genes are located on the same chromosome but at different positions. This Unigene identifier will be mapped to both Ensembl gene IDs but another external identifier mapped to only one of these 2 Ensembl gene ID won't be mapped to the other (the 
association to Hs.745351 won't be used indirectly).

An identifier scope is defined by the type of BE or probe, the source of the identifiers (database or platform) and the organism. Two scopes are different when at least one of these three elements is different. Mapping is the process to identify equivalent identifiers in two different scopes. This definition comes too late and is spread in the current version of the article. We will improve it in the next version.

Methods. The sections "Feeding the Database" and "Querying the Database" are very brief and would benefit from much more detail about the functionality of the database creation and query system. Whilst these are covered in detail in the various pieces of documentation (including some very nice working examples) there is not enough in the manuscript itself to allow the reader to assess the available functionality.

We will list the available functions (at least the most relevant ones) in the next version of the article.

\section{Use Cases.}

There appears to be a discrepancy in gene counts from the Ensembl examples used in this section; the first example calls human Ensembl genes and returns 59,515 genes the second states the total number of human Ensembl genes to be 68,460 .

59,515 corresponds to the number of BE (Gene in this case). 68,460 corresponds to the number of BEIDs (Ensemble gene IDs in this case). As explained multiple BEID can identify the same BE. In other words 59,515 BE are identified by 68,460 BEID.

Figure 3. illustrates a relationship graph including deprecated

BEIDs. Whilst is_replaced_by is clear, it is not clear (or defined anywhere) what the meaning of is_associated_to is and how that differs from corresponds_to. This should be clarified in the text.

See our answer about transitivity. We will make it clearer in the next version of the article.

The sentence "The function guessIdOrigin..." appears out of place, unconnected to the surrounding text.

We agree and it will be moved to another place in the next version of the article (probably in the "Additional features" section).

The statement "Five identifiers were only..." and the following sentence should be combined and re-worded so that the explanation as to why 5 BEIDs were uniquely found by gProfiler is clearer.

We will refine this part to address your comment and the similar one raised by the other reviewer.

No validation or commentary has been presented to test the efficacy of inferences made by the query system. I would like to have seen an attempt made to check the veracity of mappings made in this way especially when the majority (c.80\%) of extra Ensembl->EntrezID mappings recovered via BED were inferred.

This kind of validation is quite difficult. We propose to use gene coordinates provided by the NCBI and Ensembl in order to compare the position on chromosomes of genes which are mapped by the different tool. Two mapped gene identifiers should have identical or similar locations. We will add these results in the next version of the article.

A "rough approximation" of timings for queries within BED and across other 
systems is not particularly informative. It would have been straightforward to automate a sampling approach to generate a mean response time (and a variance) to a defined set of query sizes/complexities to give the user a better understanding of how variable these response times are between the systems in practice. In addition, it would have been nice to see some analysis/discussion about the "scalability" of the system as this is likely to be of particular interest to end-users considering a similar modelling approach in other domains.

We will make the analysis of mean response time with different kinds of queries and we will incorporate the results in the next version of the article. Scalability is not discussed because it highly depends on the graph database system. Here we use Neo4j for which scalability depends on the edition, community or enterprise (https://neo4j.com/subscriptions/).

Figure5. The meaning of directionality here is not clear. Whilst I can see the benefit for provenance reasons i.e. a mapping from EntrezGene to RefSeq its meaning here is somewhat moot.

This comment has also been made by the other reviewer. We will remove the arrows from the edges in figure 5 to avoid the confusion about the use of directionality to find a path between two identifiers.

Competing Interests: No competing interests were disclosed.

Reviewer Report 05 March 2018

https://doi.org/10.5256/f1000research.15138.r31026

(C) 2018 Summer-Kutmon $\mathbf{M}$ et al. This is an open access peer review report distributed under the terms of the Creative Commons Attribution License, which permits unrestricted use, distribution, and reproduction in any medium, provided the original work is properly cited.

\section{Denise Slenter}

Department of Bioinformatics - BiGCaT, Maastricht University, Maastricht, The Netherlands

Martina M. Summer-Kutmon

Department of Bioinformatics - BiGCaT, Maastricht University, Maastricht, The Netherlands

The article introduces BED a new identifier mapping tool. Using a graph database like Neo4j provides a fast way to query relationships between the biological entities and retrieve mappings of interest. The available source code is nicely documented and for bioinformaticians, setting up the database and running queries should be straight-forward.

Nevertheless, there are several major issues that we would like to comment on:

Already in the abstract, it is indicated that current tools cannot be easily customized and optimized for any specific use. It is unclear what the authors actually mean with this statement and how this is solved through BED. Further on it is also stated that current tools are generally dedicated to a particular domain, which is also true for BED. BED only focuses on gene related identifies (genes, transcripts, proteins) similar to mygene, Ensembl BioMart 
and g:Profiler.

In the introduction, three main challenges are mentioned which are addressed by BED.

(1) Integration of mappings from different resources - very relevant but the difficult question is if transitive mappings are always biological meaningful. They can also lead to conflicting statements when resources show inconsistent relationships (we have experienced this when comparing Ensembl $\rightarrow$ UniProt and UniProt $\rightarrow$ Ensembl mappings) - how are you dealing with that? We want to state that mygene is also integrating mappings from multiple resources.

(2) Mapping of deprecated identifiers - this is indeed an interesting problem when analysing older datasets and the visualization in Figure 3 can be very useful when running into such issues. While you mention that BED contains all deprecated identifiers, it is not discussed why g:Profiler has five deprecated identifiers that are not in BED (Figure 4).

(3) Mapping scope - It is not clear why the automation of mapping between different scopes needs to be done differently and how BED is solving this. Importantly, BioMarts and mygene also provide easy ways to map between the different scopes (gene - gene / gene - protein / gene - homolog).

Figure 3 - we believe that it would make sense to use two different edge styles for is_replaced_by and is_associated_to since they have very different meaning. Also check the layout (in this example, it looks like the blue node is placed over the edge from the purple to the light-purple node).

Figure 5 - what do the bold borders of nodes mean in the network? Preferred identifiers? How are those selected? Additionally, when talking about the shortest relevant path, the arrows on the edges might be misleading and confusing (since there is no path from ILMN_1220595 to Q16552 taking the directionality into account).

The authors shortly mention the neo2R package to build the database. The functionality is not discussed in detail and it is unclear why the existing $R$ package provided by Neo4j ( https://neo4j.com/developer/r/ was not used. Neo4j can also be easily queried from other programming languages. Are you planning to provide APIs in other languages that would allow the integration in tools other than R?

While the conversion rate from Ensembl to Entrez Gene is very interesting, we are missing a comparison between the tools for real research examples, e.g. selection of several datasets and mapping from probe to Ensembl identifier / Entrez Gene identifier (one of the most common use cases in R workflows). This is also mentioned under the criteria for a software tool article in F1000: “The article should provide examples of suitable input data sets and include an example of the output that can be expected from the tool and how this output should be interpreted."

Is it possible to only include edges from certain resources when performing the identifier conversion? Or do the users need to build their own database with only those selected resources?

As a final comment, we think that structure of the article is sometimes hard to follow and 
paragraphs are often not linked to each other. In the section "Converting identifiers" you state the following: "The aim of BED is to improve the efficiency of identifier conversion in a well defined context (organism, information resources of interest. . .) and not to replace biomaRt, mygene, gProfileR or other tools which provide many more features for many organisms and which should not be narrowed to this task for a complete comparison." We believe that this efficiency, especially in the context of run time, is the key advantage of this tool and this should be made more clear in the article (abstract/intro/conclusion).

Is the rationale for developing the new software tool clearly explained?

Partly

Is the description of the software tool technically sound?

Yes

Are sufficient details of the code, methods and analysis (if applicable) provided to allow replication of the software development and its use by others?

Yes

Is sufficient information provided to allow interpretation of the expected output datasets and any results generated using the tool?

Partly

Are the conclusions about the tool and its performance adequately supported by the findings presented in the article?

Partly

Competing Interests: We would like to note that the in the article mentioned BridgeDb framework is developed within our group.

We confirm that we have read this submission and believe that we have an appropriate level of expertise to confirm that it is of an acceptable scientific standard, however we have significant reservations, as outlined above.

Author Response 26 Apr 2018

Patrice Godard, UCB, Belgium

Thanks for having taken the time to review this article and for your constructive comments that will help us to improve its quality. We are working on a second version. In the mean time we would like to provide you some feedback about the different issues you arose and how we are going to take your comments into account in the second version of our manuscript. Also we would like to inform you that we are going to use an updated version of the BED instance based on version 92 of Ensembl (released in April). Thus numbers provided in the article will slightly change in the next version.

Already in the abstract, it is indicated that current tools cannot be easily customized and optimized for any specific use. It is unclear what the authors actually mean with this statement and how this is solved through BED. Further 
on it is also stated that current tools are generally dedicated to a particular domain, which is also true for BED. BED only focuses on gene related identifies (genes, transcripts, proteins) similar to mygene, Ensembl BioMart and g:Profiler. This statement has also been questioned by the other referee although slightly differently. We wanted to highlight the point that the way the mapping is done by most of these resources (excepted BridgeDB) cannot be customized, optimized or extended by the user according to his knowledge or to internal, non-public or non-standard information. These tools are dedicated to a particular domain: they are focused on species, type of identifiers, and update frequencies (as stated in BridgeDB publication by van Iersel et al. (2010)). It's convenient because ready to use but not flexible as BridgeDB or BED which allow an empowered user to focus on required information. We are going to modify this statement to make it less ambiguous in the next version.

In the introduction, three main challenges are mentioned which are addressed by BED.

(1) Integration of mappings from different resources - very relevant but the difficult question is if transitive mappings are always biological meaningful. They can also lead to conflicting statements when resources show inconsistent relationships (we have experienced this when comparing Ensembl $\rightarrow$ UniProt and UniProt $\rightarrow$ Ensembl mappings) - how are you dealing with that? We want to state that mygene is also integrating mappings from multiple resources.

The transitivity mechanism is managed by the 2 following relationships: "corresponds_to" and "is_associated_to". On one hand the "corresponds_to" relationships make the mapping transitive since 2 BEIDs connected through this kind of relationship are considered to identify the same BE. On the other hand a BEID which "is_associated_to" to another one does not automatically "identify" the same BE making this kind of relationship not available for indirect mappings. When the BED database is fed, the user chooses which relationship should be of type "corresponds_to" or of type "is_associated_to" for each resource taken into account. For example, in the instance we provide, cross references provided by Ensembl from Ensembl gene ID to Entrez, HGNC and Vega gene ID are considered as "corresponds_to" relationships whereas cross references to miRbase, Unigene and OMIM are considered as "is_associated_to" relationship. In Ensembl the Hs.745351 Unigene ID is mapped to ENSG00000184033 and to ENSG00000268651 Ensembl gene IDs which correspond to 2 different genes in Ensembl but also in Entrez and in HGNC and these genes are located on the same chromosome but at different positions. This Unigene identifier will be mapped to both Ensembl gene IDs but another external identifier mapped to only one of these 2 Ensembl gene ID won't be mapped to the other (the association to Hs.745351 won't be used indirectly).

The cross references provided by Ensembl and Uniprot between Ensembl peptide IDs and Uniprot IDs are considered as "corresponds_to" relationship in the BED instance we provide. If mygene integrates mapping from multiple resources it does not apply transitive mapping between Ensembl, Entrez and HGNC gene IDs (as shown in figure 4) and it does not allow the user to do it.

(2) Mapping of deprecated identifiers - this is indeed an interesting problem when analysing older datasets and the visualization in Figure 3 can be very useful when running into such issues. While you mention that BED contains all deprecated identifiers, it is not discussed why g:Profiler has five deprecated identifiers that are not in BED (Figure 4). 
These deprecated identifiers are not associated to any up-to-date identifier in Ensembl and as such they are not considered anymore for mapping in BED. We will develop this point in the next version of the article in order to make it clearer.

(3) Mapping scope - It is not clear why the automation of mapping between different scopes needs to be done differently and how BED is solving this. Importantly, BioMarts and mygene also provide easy ways to map between the different scopes (gene - gene / gene - protein / gene - homolog).

BED use the biological relationship between genes, transcript and peptides to convert identifiers. For example, when converting peptides identifiers from the same species it will use only mapping done at the peptide level and won't use mapping to transcript and gene mapping. This strategy seems to be applied by biomaRt but not by mygene nor by gProfileR which map for example one Uniprot ID to all the Ensembl peptide ID coded by the same gene. For example the A6NI28 Uniprot identifier is unambiguously mapped to the ENSP00000298815 Ensembl peptide identifier by BED and biomaRt but is mapped to three additional Ensembl peptide identifiers (ENSP00000431776, ENSP00000434304 and ENSP00000435961 which are encoded by the same gene: ENSG00000165895) by mygene and gProfileR. Mapping biological entities identifier which are not genes from two different organisms using ortholog information requires at least two steps in biomaRt, mygene and gProfileR: one for find the ortholog gene and the other to find the relevant biological entity identifier. These two steps are integrated and transparent in BED. We will add clarifying sentences in the next version of the article to address this.

Figure 3 - we believe that it would make sense to use two different edge styles for is_replaced_by and is_associated_to since they have very different meaning. Also check the layout (in this example, it looks like the blue node is placed over the edge from the purple to the light-purple node).

The visNetwork library only provides 2 types of edges: solid or dash. And we would prefer not using too many colors for different types of relationships. The "is_replaced_by" and "is_associated_to" relationship can easily be differentiated using the colors of the nodes: if the nodes have the same color it is an "is_replaced_by" relationship"; if the nodes have different colors it is an "is_associated_to" relationship. In this kind of graph "is_known_as", "identifies" (optional) or "targets" (optional) relationships can also be differentiated according to the shapes of the nodes. We will clarify this point in the figure legend. We will also fix the layout issue of figure 3 in the next version of the article.

Figure 5 - what do the bold borders of nodes mean in the network? Preferred identifiers? How are those selected? Additionally, when talking about the shortest relevant path, the arrows on the edges might be misleading and confusing (since there is no path from ILMN_1220595 to Q16552 taking the directionality into account).

Bold borders in this figure indeed meant preferred identifiers. In the first version of the BED instance we provided (bed-ucb-human:2018.01.03), the preferred status of RefSeq transcripts and peptides is determined according to the status field provided in the gene2refseq file provided by the NCBI. The ID is preferred if the status is "MODEL". The way to define the preferred status of Entrez gene, RefSeq transcripts and peptides will change in the next version of this instance where we will consider the assembly information also provided in the gene2refseq file: identifiers associated to non-alternative assembly will be "preferred".

We will remove the arrows from the edges in figure 5 to avoid the confusion about the use 
of directionality to find a path between two identifiers.

The authors shortly mention the neo2R package to build the database. The functionality is not discussed in detail and it is unclear why the existing $R$ package provided by Neo4j (https://neo4j.com/developer/r/) was not used. Neo4j can also be easily queried from other programming languages. Are you planning to provide APIs in other languages that would allow the integration in tools other than $\mathbf{R}$ ?

Two reasons motivated our choice to develop neo2R : (i) The development of Rneo4j package was on hold for a long time period (according to github commits) and (ii) it used legacy cypher HTTP endpoint. We wanted to use the transactional HTTP endpoint as recommended in the neo4j documentation (https://neo4j.com/docs/rest-

docs/current/\#rest-api-cypher). As the scope of the article is on the biological entity mapping and not on Neo4j as such, we don't want to put emphasis on this point because we don't think we provide strong additional value at this level.

We do not plan to provide API in other languages but we would be happy if it is done by other developers and we would be ready to help in this frame. Indeed one of the reason to make the BED package publicly available under a GPL-3 license is to allow the community to build on it and to improve it.

While the conversion rate from Ensembl to Entrez Gene is very interesting, we are missing a comparison between the tools for real research examples, e.g. selection of several datasets and mapping from probe to Ensembl identifier I Entrez Gene identifier (one of the most common use cases in $R$ workflows). This is also mentioned under the criteria for a software tool article in F1000: "The article should provide examples of suitable input data sets and include an example of the output that can be expected from the tool and how this output should be interpreted."

We will provide such an example in the next version. The example will be focused on the comparison of results from different experiments with different designs: different microarray platforms and organisms.

Is it possible to only include edges from certain resources when performing the identifier conversion? Or do the users need to build their own database with only those selected resources?

As mentioned here-above, the conversion strategy is defined when feeding the BED database and the use of the relationships: "corresponds_to" and "is_associated_to". At the end-user level, refinements of mapping can be achieved by using the "restricted" (which focus the mapping to non-deprecated identifiers) and the "preFilter" (which focus the mapping to preferred identifiers) parameters. Also the "getDirectProduct" and "getDirectOrigin" functions allow the user to find direct products or direct origins of molecular biology processes. For example the direct products of an Ensembl gene ID will be Ensembl transcript IDs. This is particularly useful when the user wants to focus on canonical transcription or translation events when this information is available (this is the case for Ensembl transcripts and peptides).

As a final comment, we think that structure of the article is sometimes hard to follow and paragraphs are often not linked to each other. In the section "Converting identifiers" you state the following: "The aim of BED is to improve the efficiency of identifier conversion in a well defined context (organism, information resources of interest. ..) and not to replace biomaRt, mygene, 
gProfileR or other tools which provide many more features for many organisms and which should not be narrowed to this task for a complete comparison." We believe that this efficiency, especially in the context of run time, is the key advantage of this tool and this should be made more clear in the article (abstract/intro/conclusion).

We adopted the structure recommended by F1000Research for a "software tool article". Nevertheless we take note of this comment and we will try to improve the flow of the text in the next version of the article.

We will put higher emphasis on the efficiency statement in the next version of the article.

Competing Interests: No competing interests were disclosed.

The benefits of publishing with F1000Research:

- Your article is published within days, with no editorial bias

- You can publish traditional articles, null/negative results, case reports, data notes and more

- The peer review process is transparent and collaborative

- Your article is indexed in PubMed after passing peer review

- Dedicated customer support at every stage

For pre-submission enquiries, contact research@f1000.com 